This item was submitted to Loughborough's Research Repository by the author.

Items in Figshare are protected by copyright, with all rights reserved, unless otherwise indicated.

\title{
An automated driver based on convergent vector fields
}

PLEASE CITE THE PUBLISHED VERSION

PUBLISHER

Professional Engineering Publishing / @ IMechE

VERSION

VoR (Version of Record)

LICENCE

CC BY-NC-ND 4.0

REPOSITORY RECORD

Gordon, T.J., Matt C. Best, and P.J. Dixon. 2009. "An Automated Driver Based on Convergent Vector Fields". figshare. https://hdl.handle.net/2134/5111. 
This item was submitted to Loughborough's Institutional Repository (https://dspace.lboro.ac.uk/) by the author and is made available under the following Creative Commons Licence conditions.

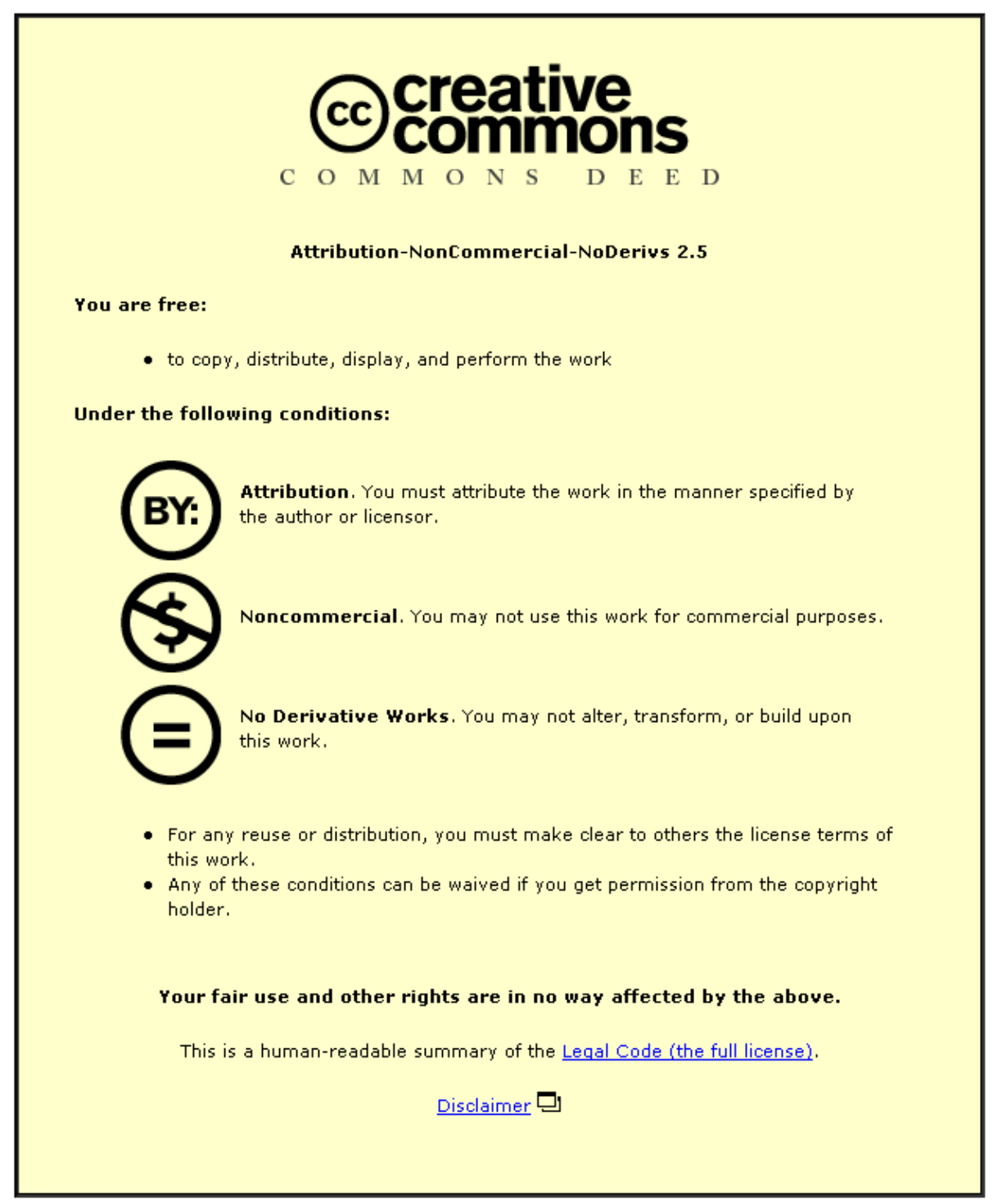

For the full text of this licence, please go to: http://creativecommons.org/licenses/by-nc-nd/2.5/ 


\title{
An automated driver based on convergent vector fields
}

\author{
T J Gordon*, M C Best and P J Dixon
}

Department of Aeronautical and Automotive Engineering, Loughborough University, Loughborough, Leicestershire, UK

\begin{abstract}
This paper describes a new general framework for the action of an automated driver (or driver model) to provide the control of longitudinal and lateral dynamics of a road vehicle. The context of the problem is assumed to be in high-speed competitive driving, as in motor racing, where the requirement is for maximum possible speed along a track, making use of a reference path (racing line) but with the capacity for obstacle avoidance and recovery from large excursions. While not necessarily representative of a human driver, the analysis provides worthwhile insight into the nature of the driving task and offers a new approach for vehicle lateral and longitudinal control; it also has applications in less demanding applications such as Advanced Cruise Control systems. As is common in the literature, the driving task is broken down into two distinct subtasks: path planning and local feedback control. In the first of these tasks, an essentially geometric approach is taken here, which makes use of a vector field analysis. At each location $\boldsymbol{x}$ the automated driver is to prescribe a vector $\boldsymbol{w}$ for the desired vehicle mass centre velocity; the spatial distribution and global properties of $\boldsymbol{w}(\boldsymbol{x})$ provide essential information for stability analysis, as well as control reference. The resulting vector field is considered in the context of limited friction and limited mass centre accelerations, leading to constraints on $\nabla \boldsymbol{w}$. Provided such constraints are satisfied, and using suitable adaptation of $\boldsymbol{w}(\boldsymbol{x})$ when required, it is shown that feedback control can be applied to guarantee stable asymptotic tracking of a reference path, even under limit handling conditions. A specific implementation of the method is included, using dual non-linear SISO (single-input single-output) controllers.
\end{abstract}

Keywords: automated driver, driver model, non-linear dynamics, vector fields, vehicle dynamics, control systems

\section{NOTATION}

Note that SI units and SAE coordinate axes are used throughout.

$a$
$\bar{a}_{\mathrm{t}}, \bar{a}_{\mathrm{n}}$
$(B, C, D, E)$
$\left(c_{1}, c_{2}\right)$
$\boldsymbol{e}(t)$
$\boldsymbol{e}_{\mathrm{t}}, \boldsymbol{e}_{\mathrm{n}}$
$\overline{\boldsymbol{e}}_{\mathrm{t}}, \overline{\boldsymbol{e}}_{\mathrm{n}}$

$a$ vehicle acceleration limit (8) tangent and normal component accelerations magic formula parameters $(0.7094,1.4097,1,0)$ cornering stiffness parameters $\left(6.88 \times 10^{4}, 7.17 \times 10^{-4}\right)$ velocity error vector unit tangent and normal to mass centre velocity unit tangent and normal to reference field

The MS was received on 6 September 2001 and was accepted after revision for publication on 21 February 2002.

* Corresponding author: Department of Aeronautical and Automotive Engineering, Loughborough University, Stewart Miller Building, Loughborough, Leicestershire LE11 3TU, UK.

$F_{x}^{\text {com }}$
$F_{x i}$
$F_{y i}$
$h$
$\mathbf{H}$
$i=1,2,3,4$
$I$
$\left(k_{1}, k_{2}\right)$
$l_{\mathrm{a}}$
$l_{\mathrm{b}}$
$l_{\mathrm{c}}$
$L$
$M$
$r, \theta$
$s, \alpha$
$s_{\max }$
$\boldsymbol{t}_{i}$
$\boldsymbol{T}^{\max }$
$\left(u_{1}^{\max }, u_{2}^{\max }\right)$

wheel force demand tractive/braking force lateral tyre force mass centre vertical height $(0.35)$ symmetric $2 \times 2$ divergence matrix vehicle corner index (front left, right, rear left, right) yaw moment of inertia (1600) control parameters $(2,2)$ mass centre distance to front axle (1.4) mass centre distance to rear axle (1.4) vehicle half-track (0.7) lookahead distance vehicle mass (1000) yaw velocity and yaw angle longitudinal slip and slip angle tyre longitudinal slip limit $(0.5)$ unit vectors in modified field construction modified field direction integrator saturation constants $(15,1)$ 


$\begin{array}{ll}U, V & \text { vehicle forward and lateral speeds } \\ \boldsymbol{v}_{\mathrm{G}} & \text { vehicle mass centre velocity } \\ \boldsymbol{w} & \text { reference vector field } \\ w_{i} & \text { vertical tyre load } \\ W^{\max } & \text { maximum engine power }\left(10^{5}\right) \\ \boldsymbol{x} & \text { spatial coordinates } \\ \beta & \text { vehicle slip angle } \\ \varepsilon & \text { residual friction } \\ \kappa & \text { flow acceleration } \\ \lambda & \text { front-rear ratio of suspension roll } \\ \mu_{1} & \text { moments }(0.5) \\ \left(\tau_{1}, \tau_{2} \tau_{3}, \tau_{4}\right) & \text { minimum divergence eigenvalue } \\ \phi, \psi, D & \text { control parameters }(0.2,0.2,0.5,0.1) \\ & \text { functions used in convergence } \\ \omega_{0} & \text { analysis } \\ & \text { yaw velocity demand }\end{array}$

\section{INTRODUCTION}

An automated (or autonomous) driver is equivalent to an aircraft autopilot - it is required to perform a control function but does not necessarily attempt to mimic the behaviour of the human driver. The term 'driver model' is also commonly used in the literature, a term that suggests an emphasis on modelling the human driver, which is not considered here. While at present there may be relatively little scope for implementing such a system in real vehicles on the highway, there is considerable interest in developing such systems to 'drive' computer models and also to gain insight into the real-world driving task. The work presented here was originally motivated by the need to 'drive' competitor vehicles in a computer game [1], where complex and semi-realistic vehicle dynamics have been implemented and automated drivers are required to control their vehicles in real-time, and in the presence of large disturbances, such as after simulated impacts with barriers and other vehicles. A closely related application is in providing an interactive environment within simulators, for driver training, design and safety evaluation of highway architecture, etc.

Early studies in this area tended to concentrate on pure steering (lateral) control and made use of linear dynamics and control methods. Weir and McRuer [2] describe the general topology of driving activity, containing feedforward ('pursuit') and feedback ('compensatory') control. In addition, they recognize the role of 'precognitive' open-loop activities, which would correspond to a steering behaviour derived from prior training or learning, e.g. in 'pre-programmed' application of opposite steering lock to control vehicle spin. The need for feedforward information in addition to feedback in driving control is well recognized by all authors in the field; this was emphasized by an early study by Tomizuka and Whitney [3] who demonstrated the use of feed- forward information in a simple human task of tracking patterns of dots on a display screen.

In the past ten years there has been considerable interest in providing more detailed models of driving behaviour, using a variety of techniques. Modjtahedzadeh and Hess [4] used classical control techniques employing separate feedforward and feedback compensators, based simply on lateral deviations from a desired path. Horiuchi and Yuhara [5] also considered pure lateral control, but with yaw angle feedback being used in addition to lateral path deviation, the method being to minimize a quadratic performance index using numerical optimization of various transfer function parameters. The above papers are typical of much of the early literature, making use of linear models and small deviations from the desired reference path. Sharp et al. [6] use linear discrete-time optimal methods for the lateral control, and again implemented feedback of path deviation and yaw rate error. A simple non-linear aspect was then introduced via the addition of saturation functions within the steering control feedback path, in order to moderate the effects of tyre force limits. This work also implemented a simple non-linear speed control policy, based on a pre-calculation of the desired braking or driving torque, as well as their distribution at the road wheels.

A more advanced approach to using optimal control was adopted by Prokop [7] who again adopted a linear quadratic method, but made use of sequential quadratic programming to implement a model predictive control strategy. In this work both longitudinal and lateral control are considered within the optimization, and the influence of constraints makes the design method inherently non-linear. Another inherently non-linear approach was presented by MacAdam and Johnson [8] who trained an artificial neural network to carry out the steering control, though limit-handling behaviour appears not to have been specifically addressed in the training and validation.

Another development was made by Plöchl and Lugner [9] who explicitly add a third level of control to deal with large disturbances; working from an otherwise linear approach, a sliding mode controller is switched on or off, depending on the size of feedback errors. A similarity will be seen in the work presented here, though it will also be clear that the starting point and methodology for the present investigation is entirely different.

All methods for driver automation presented in the literature to date are based on lumped parameter (finite state) representations of the problem. Here the feedforward or path-planning aspect is to be addressed using a distributed parameter (infinite state) representation, in the form of a reference vector field $\boldsymbol{w}(\boldsymbol{x})$. This is an alternative way to represent a finite state feedforward policy, but defining the outcome of such a policy in a spatially distributed manner-effectively considering all possible states simultaneously. The advantage will be to 
greatly simplify the control problem and provide a new perspective on the driving task, giving insights not easily obtained from earlier approaches. The approach also offers new potential for optimization, adaptation under changing conditions, as well as dealing with complex driving tasks such as navigation within moving traffic.

Section 2 introduces the underlying methodology for the vector field approach and describes a simple example of how a lookahead policy generates such a field. Some key metrics are defined and general interpretations are made. Section 3 provides a theoretical analysis and proof of key stability and convergence results, while in Section 4 a numerical study is undertaken of vector field properties, applied within a more realistic road geometry. Sections 5 and 6 then consider key issues of vehicle implementation and vehicle dynamics simulations are presented. Finally, Section 7 provides a summary and conclusions.

\section{REFERENCE VECTOR FIELD}

Consider the motion of a road vehicle in the horizontal plane. Denoting the two-dimensional coordinates of the vehicle mass centre as $\boldsymbol{x}_{\mathrm{G}}(t)$, its motion is restricted by a friction circle constraint

$$
\left|\ddot{x}_{\mathrm{G}}\right| \leqslant a
$$

where $a$ is the peak acceleration magnitude. This essentially results from the limits of tyre-road friction, and while there are many detailed factors that determine the acceleration limits, such as handling balance, braking efficiency, aerodynamics and engine power, in the 'zeroth order approximation' this appears to be a reasonable simplification for the actual performance of a highperformance vehicle under the control of an expert driver. Of course engine power limits may be very significant to competitive performance, but fundamentally should not degrade directional control and stability from the theoretical case where engine power is unbounded.

In the scenario that equation (1) is the only limit on vehicle motion, the acceleration vector may be treated as an input vector $\boldsymbol{u}(t)$, freely chosen within the magnitude constraint; this directly controls the vehicle velocity $v_{\mathrm{G}}(t)$ and the system dynamics are simply

$$
\begin{aligned}
& \dot{\boldsymbol{v}}_{\mathrm{G}}=\boldsymbol{u}(t) \\
& |\boldsymbol{u}| \leqslant a
\end{aligned}
$$

A reference input is required for $v_{\mathrm{G}}(t)$ which encodes the track information in some way, and the approach being adopted here is to define this reference as a vector field $\boldsymbol{w}(\boldsymbol{x})$ of velocities in the plane, rather than directly in terms of a reference path.

Figure 1a shows in general terms how such a field might be linked to a target path $P$, which might be a chosen racing line in competitive motor racing. Curve

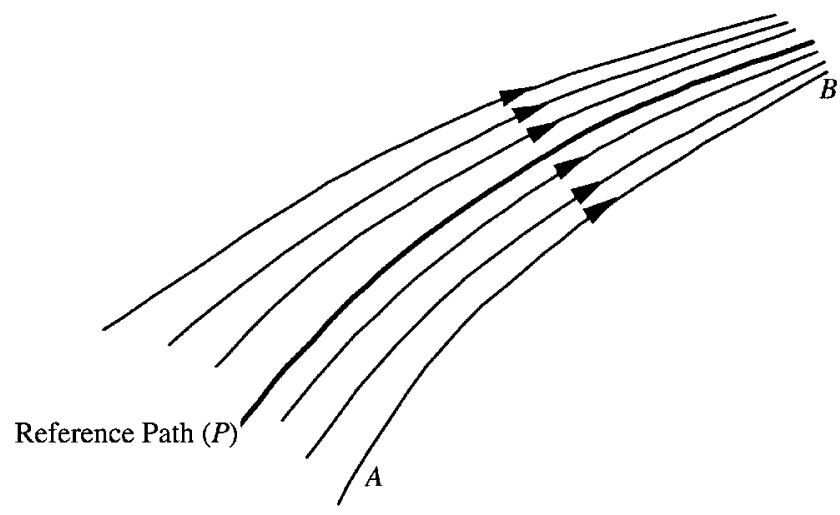

(a)

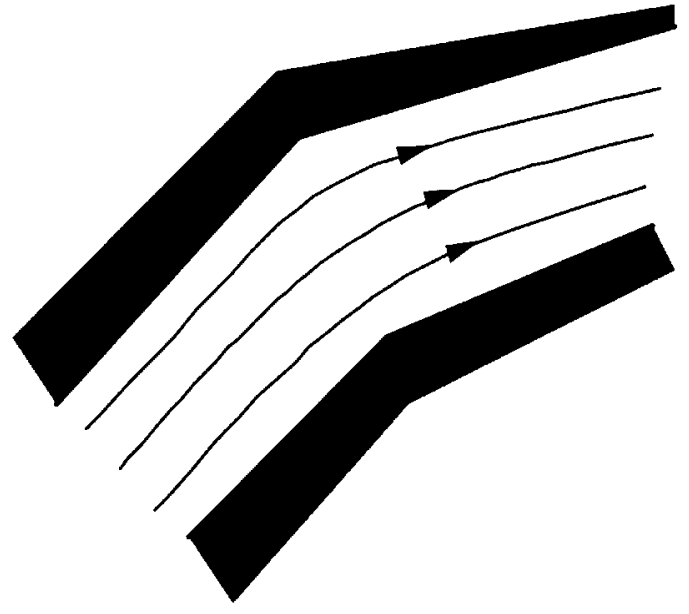

(b)

Fig. 1 General flow patterns defined by (a) a reference path and (b) track boundaries

$A B$ represents a typical integral curve ('streamline') of the reference vector field (the tangent to the curve is everywhere parallel to $\boldsymbol{w}$ ) which represents a desired recovery path back to $P$. In reality there is no absolute requirement to target a particular path and Fig. $1 \mathrm{~b}$ illustrates how a pattern of $\boldsymbol{w}$-streamlines might provide a reference for directional control without imposing convergence towards a unique vehicle path. However, in either case, it is clear that the general requirement for directional control implies a general tendency for streamlines to converge. Also note that $\boldsymbol{w}(\boldsymbol{x})$ provides a magnitude reference for vehicle speed, as well as velocity direction.

For practical purposes, $\boldsymbol{w}(\boldsymbol{x})$ may be defined via a target curve, whether or not this curve is also the desired path, and here the simple possibility illustrated in Fig. 2 is initially considered. The magnitude of $\boldsymbol{w}$ is assumed constant, and its direction is determined by point $p_{2}$, which is at a fixed lookahead distance $L$ measured along the reference curve $P$ from $p_{1}$, the point on $P$ nearest to $\boldsymbol{x}_{\mathrm{G}}$. It is easy to show that for the case where $P$ is a straight line, the integral curves exponentially decay to $P$, so that if $P$ is identified with the $x$ axis, the $\boldsymbol{w}$-stream- 


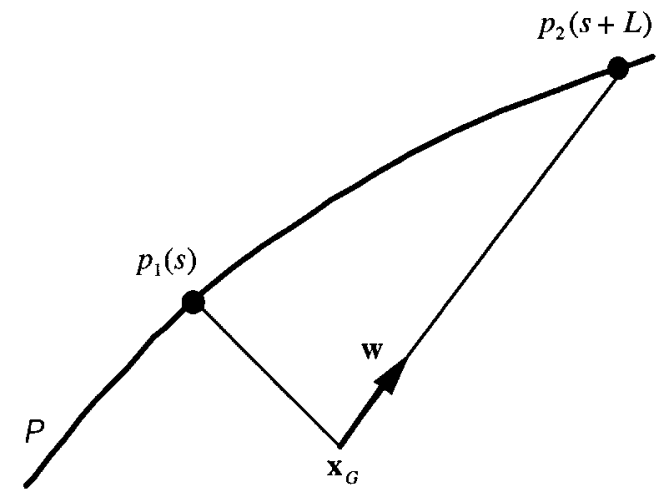

Fig. 2 Simple lookahead policy

lines in the $x y$ plane are given by (see the Appendix)

$$
y=y_{0} \exp \left[\frac{-\left(x-x_{0}\right)}{L}\right]
$$

and hence $L$ is also a relaxation length. On the other hand, if $P$ is curved, the resulting flow converges to a different path (see Section 4).

If adhering to the $w$-streamlines provides a suitable control (or navigation) strategy, and simultaneously there is control of any initial local errors, i.e.

$$
e(t)=v_{\mathrm{G}}(t)-\boldsymbol{w}\left(\boldsymbol{x}_{\mathrm{G}}(t)\right)
$$

is systematically reduced to zero, then the overall effect will be to guarantee asymptotic path following. The details will be provided in the next section, but it is immediately clear that the $\boldsymbol{w}$-streamlines must have restricted 'curvature' to allow tracking by the vehicle under the constraint of equation (2). There must also be sufficient residual friction to allow for local control of errors. Path curvature is properly quantified via the magnitude of the acceleration vector for a vehicle tracking the $\boldsymbol{w}$-streamlines without error. Clearly,

$$
\boldsymbol{a}_{\mathrm{G}}=\frac{\mathrm{d} \boldsymbol{w}}{\mathrm{d} t}=\left(w_{1} \frac{\partial \boldsymbol{w}}{\partial x}+w_{2} \frac{\partial \boldsymbol{w}}{\partial y}\right) \equiv(\boldsymbol{w} \cdot \nabla) \boldsymbol{w}
$$

where $\nabla \equiv(\partial / \partial x, \partial / \partial y)^{\mathrm{T}}$ is the two-dimensional gradient operator and $(w \cdot \nabla)$ is a scalar operator defining the time derivative of any spatial quantity due to motion along the reference field. This, taken together with the need for residual control within the friction circle, implies from equation (1) that the 'flow acceleration' is

$$
\kappa \equiv|(\boldsymbol{w} \cdot \nabla) \boldsymbol{w}| \leqslant a-\varepsilon
$$

where

$$
0<\varepsilon<a
$$

It also turns out that convergence of neighbouring streamlines provides another obstacle to asymptotic path following. This may be quantified via a suitable measure of the divergence of the flow. A common measure is the scalar divergence, which in two dimensions takes the form

$$
\nabla \cdot \boldsymbol{w}=\frac{\partial w_{1}}{\partial x}+\frac{\partial w_{2}}{\partial y}
$$

However, this simply defines the volumetric expansion of the flow and turns out to be of little relevance here. Instead a symmetric $2 \times 2$ matrix $\mathbf{H}$ with components is defined:

$$
h_{i j}=\frac{1}{2}\left(\frac{\partial w_{i}}{\partial x_{j}}+\frac{\partial w_{j}}{\partial x_{i}}\right)
$$

Being symmetric, its eigenvalues $\mu_{1}$ and $\mu_{2}$ are real, and it is an elementary result that

$$
\mu_{1}+\mu_{2}=\nabla \cdot w
$$

and hence these eigenvalues are closely related to the scalar divergence. Let $\mu_{1}$ and $\mu_{2}$ be chosen so that $\mu_{1} \leqslant \mu_{2}$; then $\mu_{1}$ represents the minimum degree of translational divergence (or, equivalently, the maximum translational convergence). More precisely (see the Appendix), if two adjacent points in the flow are separated by distance $d(t)$, then

$$
\frac{\dot{d}}{d} \geqslant \mu_{1}
$$

Figure 3 illustrates the basic point; in Fig. 3a the flow diverges, no correction is made to a vehicle path and yet a small initial error in direction of the vehicle velocity is naturally damped out: $\theta_{2}<\theta_{1}$. By contrast, in Fig. $3 \mathrm{~b}$ the flow converges and again without any correction to the velocity, errors naturally grow: $\theta_{2}>\theta_{1}$.

In the fixed lookahead scheme introduced above, the

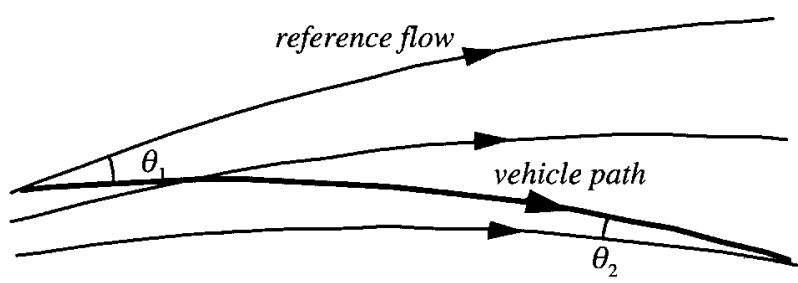

(a)

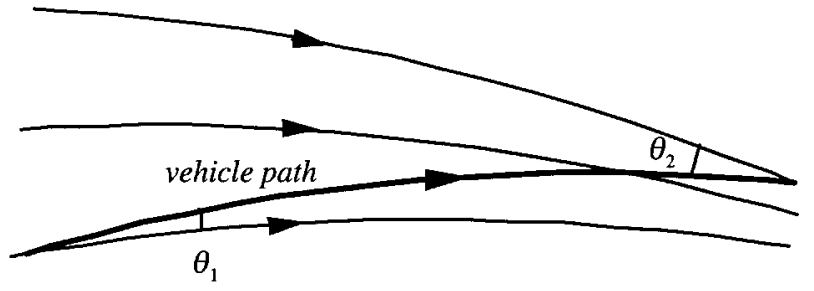

(b)

Fig. 3 Effect of the reference flow pattern on tracking error: (a) divergent flow, (b) convergent flow 
flow acceleration $\kappa$ is given by (see the Appendix)

$$
\kappa=\frac{U^{2} L y}{\left(L^{2}+y^{2}\right)^{3 / 2}}
$$

which attains a maximum value

$$
\kappa^{\max }=\frac{2 U^{2}}{3 \sqrt{3} L}
$$

As expected, this value is sensitive to vehicle reference speed and lookahead distance; to keep accelerations within bounds, a reference vector field must increase the lookahead distance in proportion to the square of the speed. Similarly, the divergence eigenvector has a minimum value

$$
\mu_{1}^{\min }=-\frac{U}{L}
$$

which is attained on the reference path itself; again the lookahead distance must increase with vehicle speed to limit the divergence of the flow. These various issues, which have been introduced quite informally in the above, are now analysed in more detail.

\section{FORMAL CONVERGENCE CRITERIA}

To be specific, it will be assumed that the vehicle is to track a predetermined path $P$, though this can be generalized to a two-dimensional target region without serious alteration to the discussion. Firstly, the problem of local error reduction towards a prescribed reference vector field $\boldsymbol{w}$ is considered in detail. Secondly, a local condition on $\boldsymbol{w}(\boldsymbol{x})$ that implies global convergence of the $w$-streamlines towards the target path $P$ is defined. Then a proof is given of what might seem intuitively obvious, that these two conditions together are sufficient to guarantee asymptotic convergence of the vehicle path to $P$.

Let $\boldsymbol{e}(t)$ be the local error between the mass centre velocity and the reference vector field, as given in equation (4). The control objective is to reduce $\boldsymbol{e}(t)$ to zero. Differentiating equation (4) gives

$$
\begin{aligned}
\dot{\boldsymbol{e}}(t) & =\dot{\boldsymbol{v}}_{\mathrm{G}}(t)-\dot{\boldsymbol{w}}(t) \\
& =\boldsymbol{u}(t)-\left(\boldsymbol{v}_{\mathrm{G}} \cdot \nabla\right) \boldsymbol{w}
\end{aligned}
$$

Expressing this in terms of $\boldsymbol{e}, \boldsymbol{w}$ and $\boldsymbol{u}$ gives

$$
\begin{aligned}
\dot{\boldsymbol{e}} & =\boldsymbol{u}-((\boldsymbol{w}+\boldsymbol{e}) \cdot \nabla) \boldsymbol{w} \\
& =\boldsymbol{u}-(\boldsymbol{w} \cdot \nabla) \boldsymbol{w}-(\boldsymbol{e} \cdot \nabla) w
\end{aligned}
$$

If $\boldsymbol{e}$ is initially zero, it can be maintained at zero via the control

$$
\boldsymbol{u}(t)=\boldsymbol{u}_{1}(\boldsymbol{x}(t)) \equiv(\boldsymbol{w} \cdot \nabla) \boldsymbol{w}
$$

which represents the effect of compensating for path curvature and longitudinal acceleration along the $\boldsymbol{w}$-stream- lines. Corresponding to equation (6),

$$
\left|\boldsymbol{u}_{1}(\boldsymbol{x})\right| \leqslant a-\varepsilon
$$

Beyond this, additional control is generally required to reduce $|\boldsymbol{e}(t)|$ from an initial positive value. Writing the control in the form 'reference plus residual',

$$
\boldsymbol{u}(t)=\boldsymbol{u}_{1}(\boldsymbol{x}(t))+\tilde{\boldsymbol{u}}(t)
$$

equation (16) takes the simplified form

$$
\dot{\boldsymbol{e}}=\tilde{\boldsymbol{u}}-(e \cdot \nabla) w
$$

and hence

$$
e e=\boldsymbol{e} \cdot \dot{\boldsymbol{e}}=\boldsymbol{e} \cdot \tilde{\boldsymbol{u}}-\boldsymbol{e} \cdot[(\boldsymbol{e} \cdot \nabla) \boldsymbol{w}]
$$

where $e=(e \cdot e)^{1 / 2}$ is the norm of $e$.

If $\tilde{\boldsymbol{u}}(t)$ is in the direction of some unit vector $\boldsymbol{n}$, it follows from equations (18) and (19) that the friction limit on the residual control is of the form

$$
\begin{aligned}
& |\tilde{\boldsymbol{u}}(t)| \leqslant M(\boldsymbol{x}, \boldsymbol{n}) \\
& M(\boldsymbol{x}, \boldsymbol{n}) \geqslant \varepsilon
\end{aligned}
$$

$M(\boldsymbol{x}, \boldsymbol{n})$ is an upper bound on the residual control and $\varepsilon$ is the least upper bound. In Fig. 4 the friction circle is of radius $a$ and a smaller circle of radius $a-\varepsilon$ is also drawn. In the limiting case the reference control $\boldsymbol{u}_{1}$ reaches the inner circle and the residual control is bounded by the outer circle. The least bound on the magnitude of $\tilde{\boldsymbol{u}}$ is therefore $\varepsilon$, while the family of vectors labelled $\tilde{\boldsymbol{u}}$ coincide with the directional dependent bound $M(\boldsymbol{x}, \boldsymbol{n})$. From the assumptions above, $\tilde{\boldsymbol{u}}(t)$ may be freely chosen within this bound, and maximal error reduction implies $\tilde{\boldsymbol{u}}(t)$ can be chosen to make the right-hand side of equation (21) as negative as possible, which is achieved by the non-linear residual control:

$$
\tilde{\boldsymbol{u}}(t)= \begin{cases}-M(\boldsymbol{x},-\hat{\boldsymbol{e}}) \hat{\boldsymbol{e}} & \text { if }|\mathbf{e}|>v_{0} \\ -\varepsilon v_{0} \boldsymbol{e} & \text { otherwise }\end{cases}
$$

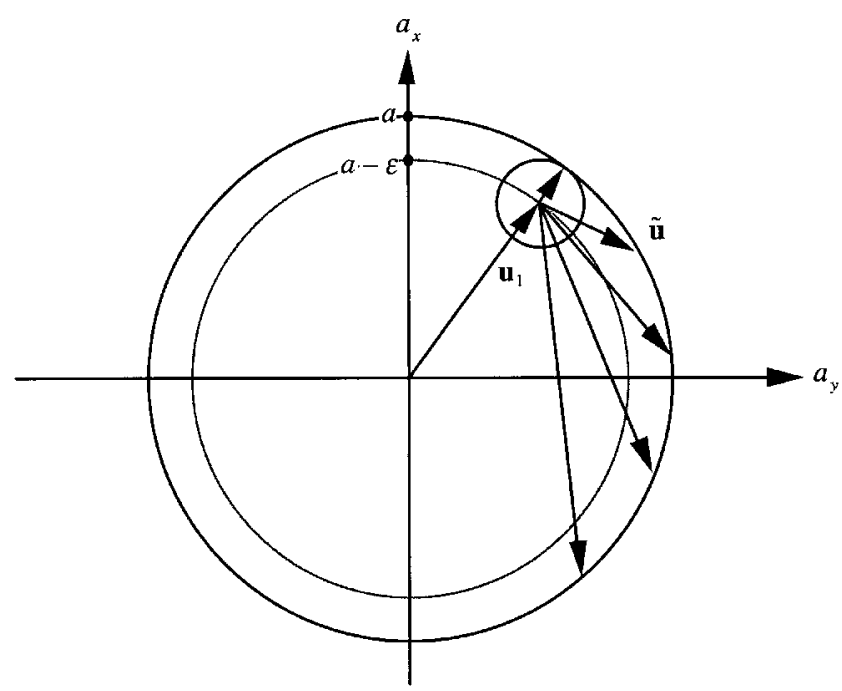

Fig. 4 Use of the friction circle 
Here $\hat{\boldsymbol{e}}$ is a unit vector in the direction of $\boldsymbol{e}$ and $v_{0}$ is a small velocity magnitude required to smoothly reduce the residual control to zero very close to $\boldsymbol{e}=\mathbf{0}$. Applying this in equation (21) gives

$$
e \dot{e}=-M(\boldsymbol{x},-\hat{\boldsymbol{e}}) e-\boldsymbol{e} \cdot[(\boldsymbol{e} \cdot \nabla) \boldsymbol{w}]
$$

The second term may be rewritten more transparently via the divergence matrix $\mathbf{H}$ of equation (9) to give

$$
e e=-M(\boldsymbol{x},-\hat{\boldsymbol{e}}) e-\boldsymbol{e}^{\mathrm{T}} \mathbf{H} \boldsymbol{e}
$$

The final term is then bounded by the smallest eigenvalue of $\mathbf{H}$ to give

$$
e \dot{e} \leqslant-M(\boldsymbol{x},-\hat{\boldsymbol{e}}) e-\mu_{1} e^{2}
$$

and using equations (22) the following lower bound is finally obtained on the increase of the error norm:

$$
\dot{e} \leqslant-\varepsilon-\mu_{1} e
$$

It is clear from this that if $\mu_{1} \geqslant 0$, i.e. there is no convergence in the flow, then

$$
\dot{e} \leqslant-\varepsilon
$$

whenever $e>0$ and hence, in the absence of additional perturbations, an initial error $e_{0}>0$ is reduced to zero in a finite time:

$$
t_{0}=\frac{e_{0}}{\varepsilon}
$$

If the flow is strictly divergent, $\mu_{1}>0$, then even without residual friction or feedback control

$$
\dot{e} \leqslant-\mu_{1} e
$$

and hence

$$
e(t) \leqslant e_{0} \exp \left(-\mu_{1} t\right)
$$

and initial errors naturally decay to zero. This is in agreement with the earlier informal description illustrated in Fig. 3.

Of course, the most relevant and significant situation is when the flow is convergent, $\mu_{1}<0$, and initial errors are present. Let $\mu_{1}^{\min }<0$ be a lower bound for the eigenvalue and suppose by comparison with condition (28) that the following condition is to be imposed:

$$
\dot{e} \leqslant-\frac{1}{2} \varepsilon
$$

This will again guarantee a finite-time decay of errors, but from condition (27)

$$
\left|\mu_{1}^{\min }\right| e_{0} \leqslant \frac{1}{2} \varepsilon
$$

or

$$
e_{0} \leqslant \frac{\varepsilon}{2\left|\mu_{1}^{\min }\right|}
$$

is required. Thus flow convergence and limited residual friction conspire to restrict the size of initial errors $e_{0}$, which may be guaranteed to be damped out via control action. This is a fundamental point, which clearly implies that path planning may not be fully decoupled from the feedback control of vehicle states; when initial errors are large, some form of flow adaptation is needed to either reduce acceleration (reduce $\kappa$ and hence increase $\varepsilon$ ) or reduce the flow convergence (hence reducing $\left|\mu_{1}^{\min }\right|$ ) or of course both together. While the issue of field adaptation will not be considered in any detail, it will be assumed that initial errors are small enough to satisfy condition (34) or equivalently that a suitable adaptation has already been carried out; then condition (32) is valid.

The finite time error decay implied by condition (32) results specifically from the use of non-linear feedback and assumes that no further disturbances arise. Hence it seems desirable to weaken this condition for the general analysis and assume instead that $e(t)$ is bounded by a smooth positive function $\psi(t)$ :

$$
e(t) \leqslant \psi(t)
$$

which satisfies

$$
\psi(t) \rightarrow 0 \quad \text { as } t \rightarrow \infty
$$

The convergence analysis may now be completed, by defining a condition for the globally convergent structure of the $w$-streamlines towards $P$. This can be formulated via a smooth distance function $D(\boldsymbol{x})$, defined in some domain $\Omega$ containing $P$ :

$$
\begin{array}{ll}
D(\boldsymbol{x}) \geqslant 0 & \text { if } \boldsymbol{x} \in \Omega \\
D(\boldsymbol{x})=0 & \text { if and only if } \boldsymbol{x} \in P
\end{array}
$$

$D(\boldsymbol{x})$ is to be defined so that the gradient function $\nabla D$ is bounded, and without loss of generality it is assumed that

$$
|\nabla D(\boldsymbol{x})| \leqslant 1 \quad \text { for all } \boldsymbol{x} \in \Omega
$$

The global requirement that $\boldsymbol{w}$ converges towards the target path is easily expressed by the condition that the distance function $D$ decreases monotonically along its integral curves, which is a simple local requirement, as follows:

$$
(w(x) \cdot \nabla) D(x) \leqslant-\phi(D(x))
$$

for all $\boldsymbol{x} \in \Omega$. Here $\phi$ is a smooth positive-definite function:

$$
\begin{array}{ll}
\phi(D)>0 & \text { for all } D>0 \\
\phi(0)=0, & \phi^{\prime}(D) \geqslant 0
\end{array}
$$

The asymptotic path following can now be characterized by the condition

$$
D\left(x_{\mathrm{G}}(t)\right) \rightarrow 0 \quad \text { as } t \rightarrow \infty
$$

Writing $D(t)$ as a shorthand for $D\left(x_{\mathrm{G}}(t)\right)$ gives

$$
\begin{aligned}
\dot{D}(t) & =\left(\boldsymbol{v}_{\mathrm{G}} \cdot \nabla\right) D \\
& =((\boldsymbol{w}+\boldsymbol{e}) \cdot \nabla) D \\
& =(\boldsymbol{w} \cdot \nabla) D+(\boldsymbol{e} \cdot \nabla) D \\
& \leqslant-\phi(D)+|(\boldsymbol{e} \cdot \nabla) D|
\end{aligned}
$$


The second term is simply the norm of the scalar product between the vectors $\boldsymbol{e}$ and $\nabla D$, which is therefore bounded by the product of the vector norms:

$$
\begin{aligned}
\dot{D}(t) & \leqslant-\phi(D)+e(t)|\nabla D| \\
& \leqslant-\phi(D)+e(t)
\end{aligned}
$$

with the last step following from condition (38). Hence, from condition (35)

$$
\dot{D}(t) \leqslant-\phi(D)+\psi(t)
$$

which is a simple scalar inequality that can be shown to imply $D(t) \rightarrow 0$ as $t \rightarrow \infty$ as required (see the Appendix).

The major purpose of this section has now been achieved and can be summarized as follows. Given:

(a) a vector field structure converging to a path $P$,

(b) full control of mass centre accelerations within the friction circle,

(c) flow accelerations that remain within suitable bounds and

(d) flow convergence limited by maximum assumed initial velocity errors,

then the vehicle path is guaranteed to converge to $P$.

\section{FIELD CONSTRUCTION AND THE IDEAL VEHICLE RESPONSE}

Following on from the analytical investigation of Section 3, a numerical approach is now applied to a more realistic though simple track geometry shown in Fig. 5. This is based on a centre-line comprising two quarter-circle arcs, each of radius $50 \mathrm{~m}$, and with nom-

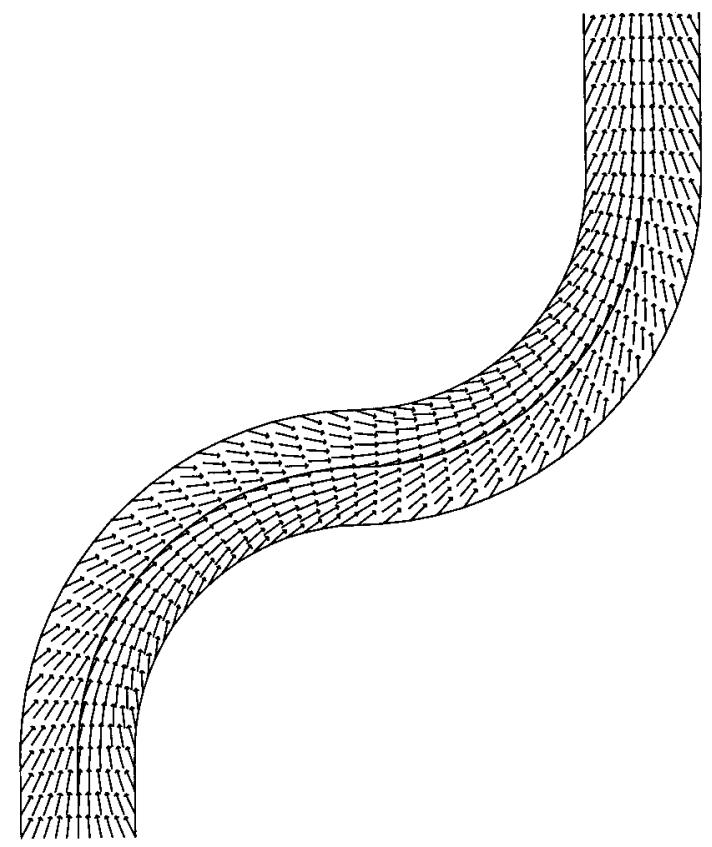

Fig. 5 Reference vector field on a curved track inal track boundaries displaced $\pm 10 \mathrm{~m}$ laterally; each arc is connected to entry and exit straights as shown. A simple reference vector field is also shown, this being derived from a constant lookahead distance of $20 \mathrm{~m}$, using the centre-line as a target path. Initially, a constant reference speed of $20 \mathrm{~m} / \mathrm{s}$ is assumed, so that the maximum lateral acceleration on the centre-line is $8 \mathrm{~m} / \mathrm{s}^{2}$. Clearly, on the curved portions of the track, the reference field is not tangential to the centre-line, so this is not the 'intended' vehicle path.

Figure 6 maps the integral curves of the flow field, which physically corresponds to vehicle paths that follow the reference vector field, starting at various points on the track; the track centre-line is also shown. Here there are no friction circle constraints imposed, so a vehicle following these curves essentially achieves perfect tracking. As expected from Fig. 5, the flow converges to a path that 'cuts inside' the track centre-line. Figure 7 shows a more complex set of dynamic responses, where a 'vehicle friction' limit $a=8 \mathrm{~m} / \mathrm{s}^{2}$ is imposed. The dynamics described in Sections 2 and 3 have been imposed, so this is essentially a friction-limited particle model of the vehicle, with feedback control prescribed by equation (23). The initial velocities are parallel to the straight section of the track, at $20 \mathrm{~m} / \mathrm{s}$, so there are initial errors as well as friction limits to contend with. Clearly the responses from entering at the left side of the track are satisfactory, while there are significant excursions apparent for right-side entry. Intuitively this should seem reasonable, and it is fundamentally due to the limitation of the simple fixed lookahead construction, which gives 'hot spots' in the flow acceleration $\kappa$ of equation (6); this is mapped out in Fig. 8. The four shadings correspond to flow acceleration ranges: $\left(0-4 \mathrm{~m} / \mathrm{s}^{2}\right)$,

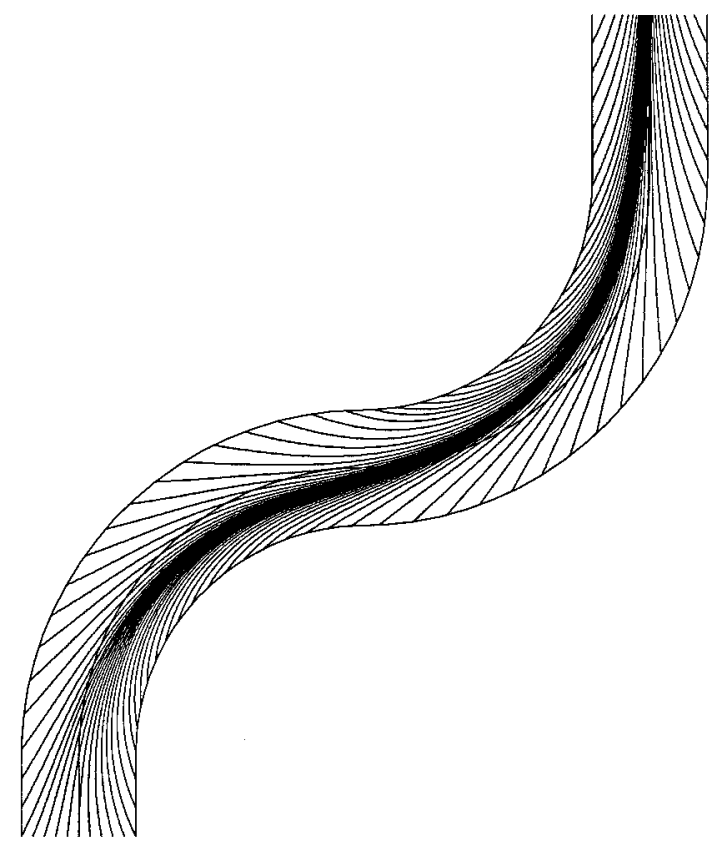

Fig. 6 Integral curves based on a constant lookahead distance 


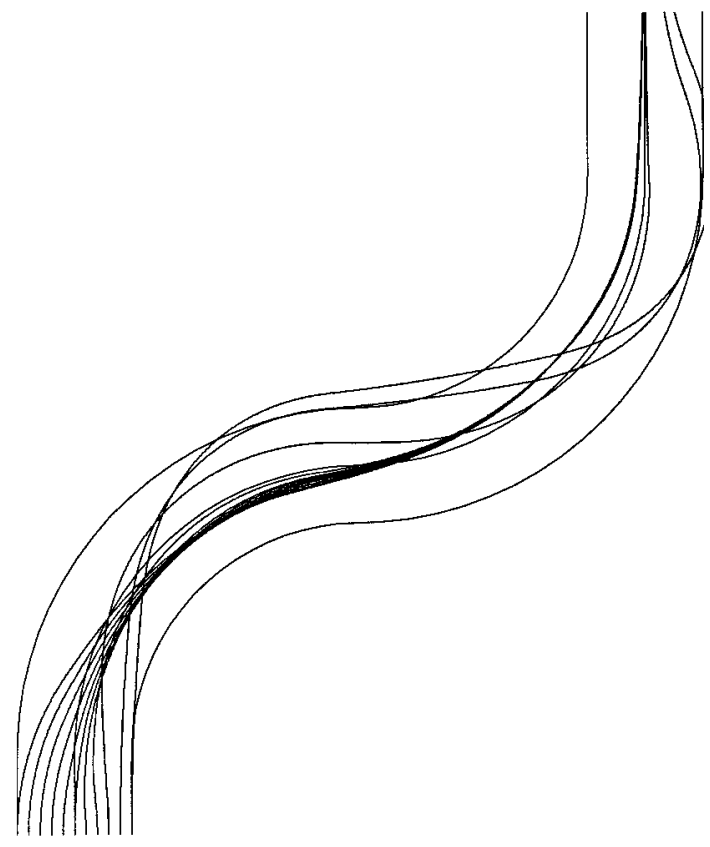

Fig. 7 Friction-limited paths for the ideal vehicle

$\left(4-8 \mathrm{~m} / \mathrm{s}^{2}\right),\left(8-12 \mathrm{~m} / \mathrm{s}^{2}\right),\left(12-\mathrm{m} / \mathrm{s}^{2}\right)$, with low values darkest, so it is clear that equation (6) is violated in regions on the inside of the two curves. Entering the first curve from left of centre is easily controlled, while entering right of centre immediately causes excessive demands on the simplified vehicle, and explains why a vehicle entering from the extreme right appears to drive straight on, regardless of the curve.

The other key variable for convergent control is $\mu_{1}$, which is mapped in Fig. 9, and this shows no similar 'hotspots'. It is reasonably uniform across the region, taking values between 0.6 and 1.0 , with the maximum values tending towards the centre of the track. This is in agreement with equation (14), from which values of order 1 would be expected, so it is clear that path curvature has little effect in this case.

The main point from the above brief example is that even before considering the details of specific vehicle dynamics, friction limits and vector field structure offer insight into the viability and relative stability of different path planning strategies. While it is certainly not the intention here to consider formal optimization of the vector field, a couple of possible 'improvements' to the basic constant lookahead strategy will be looked at before going on to investigate the applicability of the general approach to more realistic vehicle behaviour.

Firstly, it is fairly obvious from Fig. 8 that even with the fixed lookahead policy, faster cornering is possible; the vehicle accelerations at constant speed are below the vehicle limits, especially around the point where the curvature changes direction. Hence a variable reference speed is introduced along the convergent path. Any attempt to formulate and achieve this inevitably leads to some form of optimization, though in fact it is possible to obtain optimal results via a simple and direct approach. Using the same directional information as before (based on a fixed $20 \mathrm{~m}$ lookahead distance), the method is simply to increase the target speed gradually until combined lateral and longitudinal accelerations attain the assumed friction limit of $8 \mathrm{~m} / \mathrm{s}^{2}$ on the convergent path. Linear interpolation of speeds is assumed,
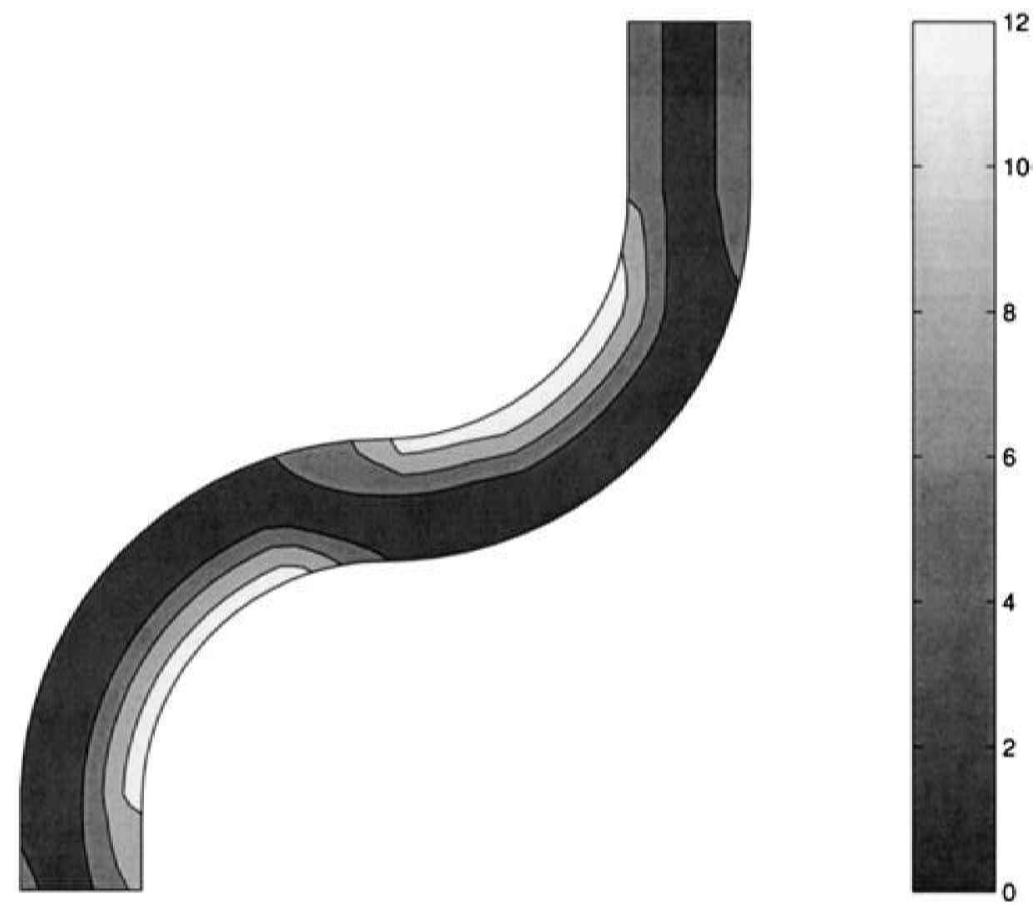

Fig. 8 Flow acceleration contours 

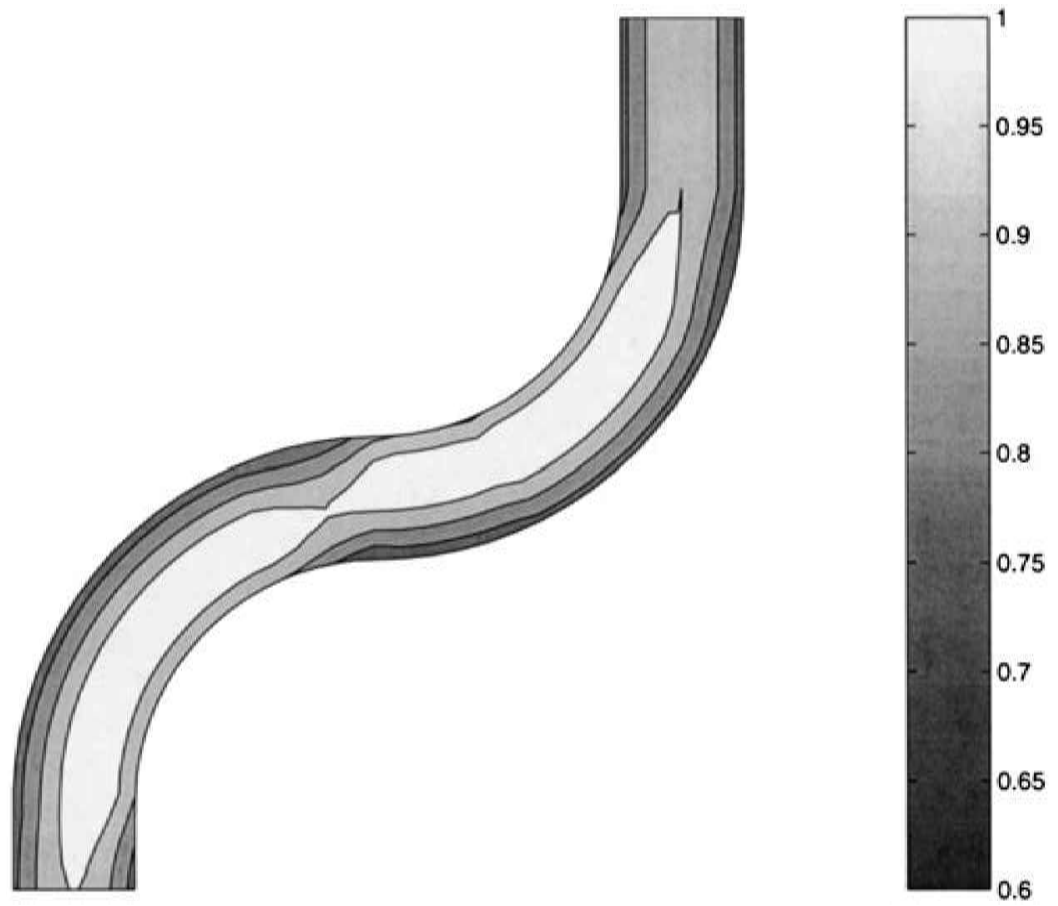

Fig. 9 Magnitude of the minimum divergence eigenvalue

sampling at $4 \mathrm{~m}$ distances along the track centre-line. At each iteration the speed is increased by a fixed step provided the acceleration constraint is satisfied throughout both neighbouring track segments. Eventually, when no further increases are possible, the overall maximum acceleration is found, and if this exceeds the limit all velocities are rescaled accordingly. Once this process converges, the velocity step is reduced and the whole process repeated, and so on until satisfactory resolution is obtained. In practice this is both quick and simple (though not necessarily practical for real-time calculation on a vehicle!).

The resulting speed on the convergent path of the double-curved track is given in Fig. 10. The optimum variable speed involves braking from an initial $26 \mathrm{~m} / \mathrm{s}$, then a brief period of acceleration as the direction of curvature changes, then further braking, which is followed by a steadily increasing acceleration on to the exit straight. From this, the time to traverse the same convergent path along the track reduces to $9.1 \mathrm{~s}$ from the previous value of $9.8 \mathrm{~s}$. However, on varying the initial lateral position on the track in Fig. 11 there is clearly a much greater sensitivity than previously (Fig. 7); all the right-hand entry points give rise to large subsequent excursions, while those on the left remain well behaved. This is hardly surprising, however, since the reference path is already very close to the theoretical friction limit, and the simple expedient has been taken of interpolating to the nearest track centre point to estimate target speeds across the track. The general inference here is that speed optimization has provoked an increased sensitivity but that the overall trend is exactly as before: drifting the entry point slightly to the left is stable and acceptable to the automated (or real!) driver, while drifting to the right is undesirable. It can easily be hypothesized that a full and naõve optimization of the problem will be relatively unstable for the driver, with the entry point of the convergent path starting well left of the track centre. Of course, a very simple way to increase mean speed would be to increase the lookahead distance; provided the reference speed on the convergent path is adjusted to ensure that the flow acceleration constraint is preserved, and provided this path lies within the track boundary, such a change would be beneficial.

For a second 'improvement', it is noted that, in the above, the track centre-line has been used as something that is convenient for construction of the vector field, but not for the purpose of accurate tracking. A simple modification greatly improves the tracking of the centreline, or indeed any form of intended trajectory. Referring back to Fig. 2, let $\boldsymbol{t}_{1}$ and $\boldsymbol{t}_{2}$ be the unit tangent vectors to the target path $P$ at locations $p_{1}$ and $p_{2}$ respectively; let $\boldsymbol{t}_{3}$ be a third unit vector, pointing from $\boldsymbol{x}_{\mathrm{G}}$ to $p_{2}$. A modified field direction may then be defined by the vector

$$
T=\boldsymbol{t}_{3}+\frac{\boldsymbol{t}_{1}-\boldsymbol{t}_{2}}{2} \cos \theta
$$

where $\cos 2 \theta=\left(\boldsymbol{t}_{1} \cdot \boldsymbol{t}_{2}\right)$ defines the angle between the two tangents on $P$; this is of course provided the arc between $p_{1}$ and $p_{2}$ turns by less than $180^{\circ}$, which is surely reasonable! In this case, simple geometry shows that whenever $\boldsymbol{x}_{\mathrm{G}}$ coincides with $P$ (and hence also with $p_{1}$ ), vector $\boldsymbol{T}$ 


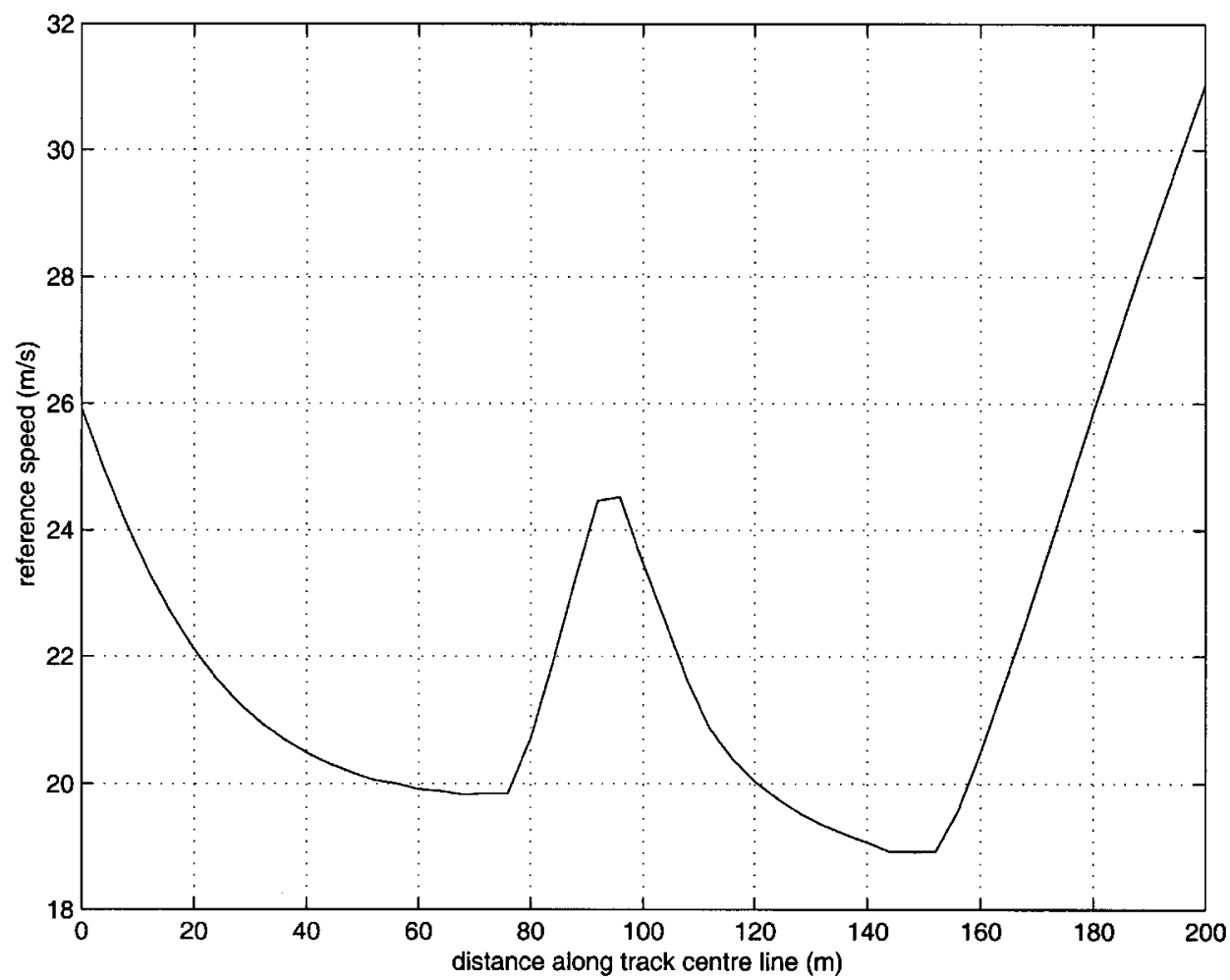

Fig. 10 Variable reference speed

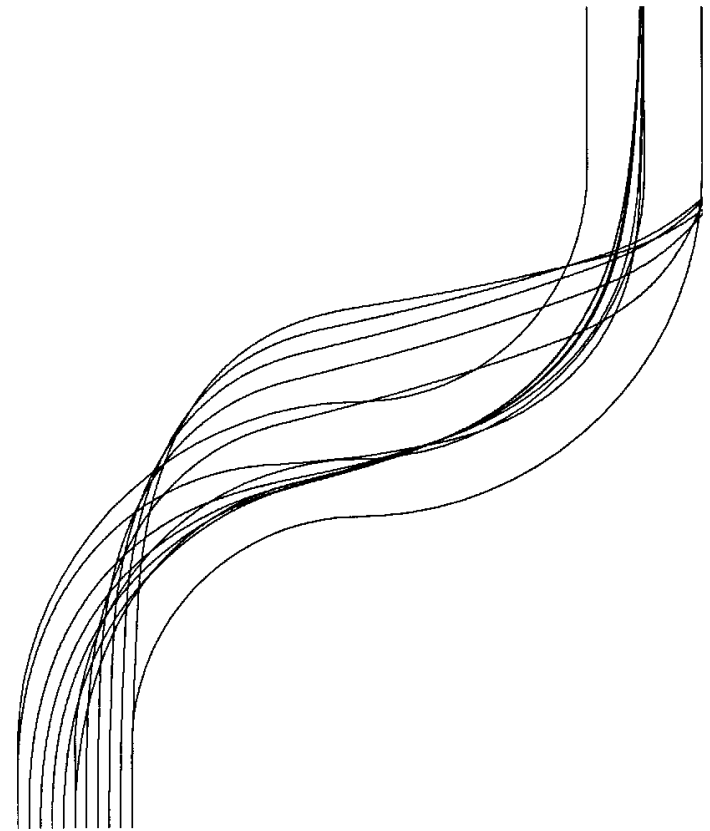

Fig. 11 Ideal vehicle paths under variable speed control

is also tangent to the arc. The general interpretation is simple; if $P$ is a straight line, the second term in equation (43) is zero and the previous form of a simple fixed lookahead is recovered; otherwise the second term provides a correction depending on the path curvature. Interestingly enough, the formula does not depend explicitly on $L$. Finally, for implementation it is neces-

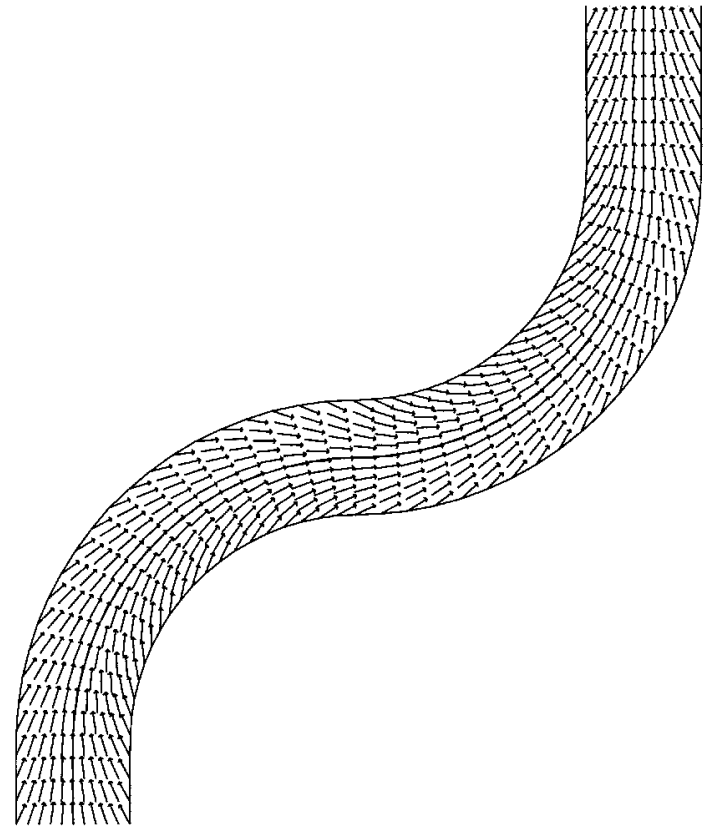

Fig. 12 Reference field modified for improved path tracking

sary to normalize and rescale:

$$
\boldsymbol{w}=\bar{v}|\boldsymbol{T}|^{-1} \boldsymbol{T}
$$

where $\bar{v}$ is the reference speed.

Figure 12 shows the flow field which results from applying this modification to the earlier example. As can be seen, the field tracks the centre-line very accurately 

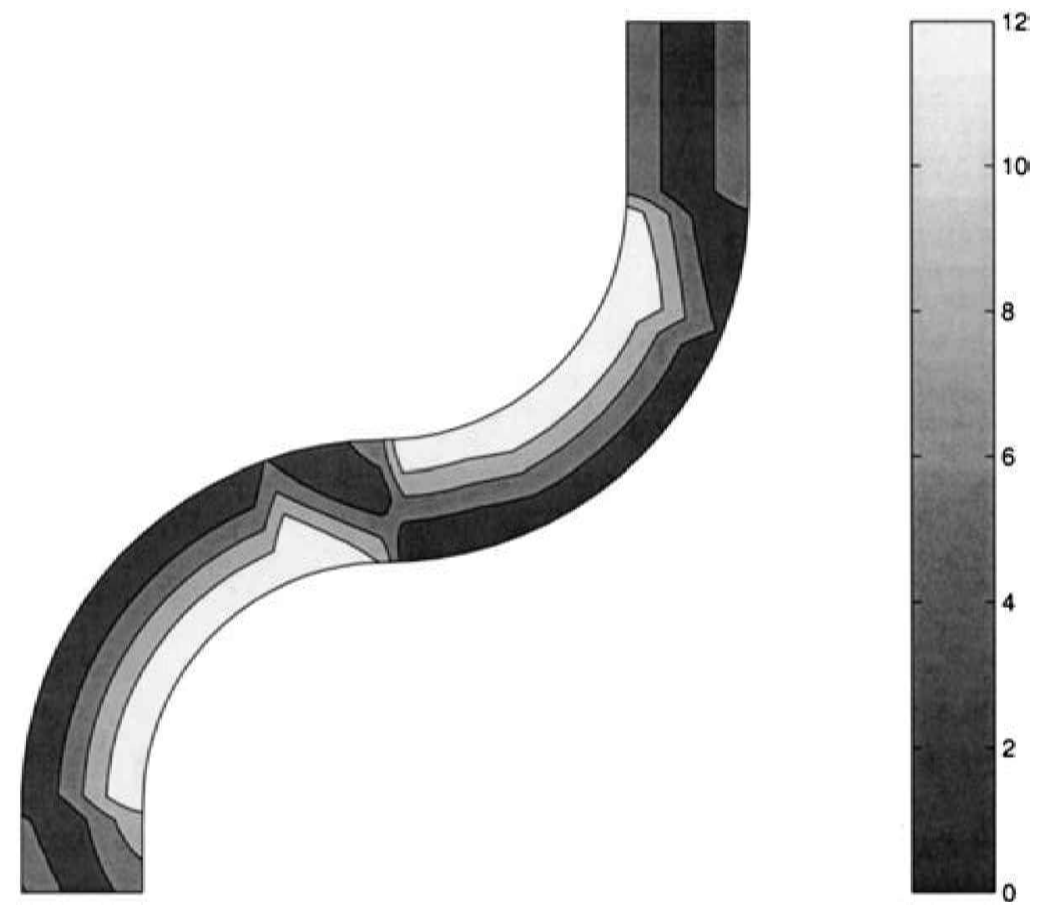

Fig. 13 Flow acceleration contours for a modified reference field

in comparison to Fig. 5, though precise tracking is not guaranteed just prior to the sudden transitions in curvature. Figure 13 maps the resulting flow accelerations, which has the same shading scale as Fig. 8. While both assume a constant speed of $20 \mathrm{~m} / \mathrm{s}$, the more accurate tracking clearly leads to increased flow accelerations in Fig. 13, especially just prior to the transition points. Of course, given the more accurate tracking property it would be feasible to increase the lookahead distance used in the construction, and also blend the transitions, but this again leads to the wider issues of optimization and will not be considered further here.

In the next section more realistic vehicle behaviour is considered as well as the question of how effectively a reference vector field can provide all of the necessary feedforward information for autonomous vehicle control.

\section{VEHICLE DYNAMICS AND CONTROLLER SYNTHESIS}

To translate desired mass centre accelerations into real life, the detailed application of vehicle controls steering, brakes and powertrain-needs to be considered. In reality this can become a complex area, involving driver learning of vehicle response characteristics, the application of chassis controls such as antilock braking and traction control, as well as suspension characteristics such as bump-steer and camber control. There is therefore a danger of losing insight because of a large amount of detail. The emphasis will be on provid- ing a relatively simple control implementation in the context of a relatively simple vehicle model, while at the same time maintaining some essential realism in the form of vehicle transient response and non-linearities, particularly in the generation of tyre forces.

Starting with the controller, reference information from the vector field $\boldsymbol{w}$ comes in the form of its magnitude $\bar{v}=|\boldsymbol{w}|$ and direction $\tan \bar{\phi}=w_{y} / w_{x}$, and also via the tangential and normal components of the flow acceleration:

$$
\bar{a}_{\mathrm{t}}=\overline{\boldsymbol{e}}_{\mathrm{t}} \cdot(\boldsymbol{w} \cdot \nabla) w, \quad \bar{a}_{\mathrm{n}}=\overline{\boldsymbol{e}}_{\mathrm{n}} \cdot(\boldsymbol{w} \cdot \nabla) \boldsymbol{w}
$$

where $\overline{\boldsymbol{e}}_{\mathrm{t}}$ and $\overline{\boldsymbol{e}}_{\mathrm{n}}$ are unit vectors tangential and normal to the reference field. Writing $\boldsymbol{e}_{\mathrm{t}}$ and $\boldsymbol{e}_{\mathrm{n}}$ for the corresponding vectors for the vehicle mass centre velocity gives

$$
\begin{aligned}
& \boldsymbol{v}_{\mathrm{G}}=v_{\mathrm{G}} \boldsymbol{e}_{\mathrm{t}} \\
& \boldsymbol{a}_{\mathrm{G}}=\dot{v}_{\mathrm{G}} \boldsymbol{e}_{\mathrm{t}}+v_{\mathrm{G}} \dot{\boldsymbol{\phi}} \boldsymbol{e}_{\mathrm{n}}
\end{aligned}
$$

Relative to vehicle coordinates, $\boldsymbol{v}_{\mathrm{G}}$ has longitudinal and lateral components $U, V$ respectively.

Longitudinal control is executed via an acceleration command signal $u_{1}(t)$, which is easily converted to a commanded drive force or braking torque delivered to the tyres. The control is determined via a forward acceleration demand

$$
a_{0}(t)=\bar{a}_{\mathrm{t}}=\frac{\bar{v}-v_{\mathrm{G}}}{\tau_{1}}
$$


and feedback of longitudinal vehicle acceleration

$$
\dot{u}_{1}=\frac{a_{0}-U}{\tau_{2}}
$$

The demand signal (48) is a natural sum of reference plus feedback and $\tau_{1}$ is a control parameter in the form of an assumed time constant. Equation (49) is essentially an integral control, and while $\dot{U}$ is not strictly the forward vehicle acceleration, the error is small provided the lateral velocity $V$ is small compared to $U$.

Lateral control is of course provided via the steering angle $u_{2}(t)$, and this is computed using a similar integral approach to $u_{1}(t)$, though here (angular) displacement errors are also included in the feedback. The vehicle yaw angle $\theta$ and slip angle

$$
\beta=\tan ^{-1}\left(\frac{V}{U}\right)
$$

together determine the direction $\phi$ of $\boldsymbol{v}_{\mathrm{G}}$ :

$$
\phi=\theta+\beta
$$

The reference angular velocity of $v_{\mathrm{G}}$ is given by

$$
\bar{\omega}=\frac{v_{\mathrm{G}} \bar{a}_{\mathrm{n}}}{\bar{v}^{2}}
$$

This is then combined with angular error to provide an angular velocity demand:

$$
\omega_{0}(t)=\bar{\omega}-\frac{\phi-\bar{\phi}}{\tau_{3}}
$$

which is translated into steering control via

$$
\dot{u}_{2}=k_{1}\left(\omega_{0}-r\right)
$$

where $r=\theta$ is the vehicle yaw rate. A simple but important point is that equation (54) is much preferred to the more 'correct' looking control equation:

$$
\dot{u}_{2}=k_{1}\left(\omega_{0}-\dot{\phi}\right)
$$

This is because steering control has a much more direct effect on yaw rate than on the angular velocity of $v_{\mathrm{G}}$, and near the limits of friction equation (55) can excite large excursions in vehicle slip angle. For the same reason, an improved equation can incorporate additional explicit control of vehicle slip angle:

$$
\dot{u}_{2}=k_{1}\left(\omega_{0}-r\right)+k_{2} \sigma(\beta \beta) \beta
$$

where $\sigma($.$) is a switching term:$

$$
\sigma(u)= \begin{cases}1 & \text { if } u>0 \\ 0 & \text { if } u \leqslant 0\end{cases}
$$

One final aspect of the controller is that to avoid excessive windup, both integrators, (49) and (56), are required to saturate at suitably high limits:

$$
\dot{u}_{i} \rightarrow 0 \quad \text { when } u_{i} \dot{u}_{i}>0 \text { and }\left|u_{i}\right|>u_{i}^{\max }
$$

The vehicle simulation model is also chosen to be reasonably simple, and yet it is important for it to incorporate a number of realistic features, especially the nonlinear properties of tyres and load transfer. Hence a three-degree-of-freedom model is used for the vehicle body. Longitudinal and lateral load transfer effects are included, though suspension travel is assumed to be minimal. The equations of motion are as follows:

$$
\begin{aligned}
& M(\dot{U}-V r)=\sum F_{x i} \\
& M(\dot{V}+U r)=\sum F_{y i} \\
& I \dot{r}=N \boldsymbol{F}
\end{aligned}
$$

Here

$$
\boldsymbol{F}=\left[F_{x 1}, F_{x 2}, F_{x 3}, F_{x 4}, F_{y 1}, F_{y 2}, F_{y 3}, F_{y 4},\right]^{\mathrm{T}}
$$

is an eight-component vector of longitudinal and lateral forces and

$$
N=\left[\begin{array}{llllllll}
l_{\mathrm{c}} & -l_{\mathrm{c}} & l_{\mathrm{c}} & -l_{\mathrm{c}} & l_{\mathrm{a}} & l_{\mathrm{a}} & -l_{\mathrm{b}} & -l_{\mathrm{b}}
\end{array}\right]
$$

is an array of coefficients used to define the yaw moment, where $l_{\mathrm{a}}+l_{\mathrm{b}}$ is the wheelbase and $2 l_{\mathrm{c}}$ is the lateral track. Assuming a fixed ratio $\lambda$ between the front and rear suspension roll moments, the vertical loads vary dynamically according to the following equation:

$$
\left[\begin{array}{cccc}
1 & 1 & 1 & 1 \\
-l_{\mathrm{a}} & -l_{\mathrm{a}} & l_{\mathrm{b}} & l_{\mathrm{b}} \\
l_{\mathrm{c}} & -l_{\mathrm{c}} & l_{\mathrm{c}} & -l_{\mathrm{c}} \\
1 & -1 & -\lambda & \lambda
\end{array}\right]\left[\begin{array}{c}
w_{1} \\
w_{2} \\
w_{3} \\
w_{4}
\end{array}\right]=\left[\begin{array}{c}
M g \\
h \sum F_{x i} \\
h \sum F_{y i} \\
0
\end{array}\right]
$$

where $h$ is the height of the mass centre above ground.

The tyre model is of a simple isotropic form, based on the Pacejka Magic Formula [10]:

$$
\begin{aligned}
& P(\alpha ; B, C, D, E) \\
& \quad \equiv D \sin \left\{C \tan ^{-1}\left[B \alpha-E\left(B \alpha-\tan ^{-1} B \alpha\right)\right]\right\}
\end{aligned}
$$

The friction circle at each tyre contact patch is defined by the following simple analytic function of vertical load $w$ :

$$
\sqrt{F_{x}^{2}+F_{y}^{2}} \leqslant F_{p}=\frac{w}{1+(2 w / M g)^{3}}
$$

and the load-dependent cornering stiffness for each tyre is

$$
C_{\alpha}(w)=c_{1}\left(1-\mathrm{e}^{-c_{2} w}\right)
$$

In terms of the longitudinal slip $s$ and tyre slip angle $\alpha$, the combined slip vector is

$$
\boldsymbol{k}=\left(\begin{array}{l}
k_{x} \\
k_{y}
\end{array}\right)=\frac{C_{\alpha}}{F_{p}}\left(\begin{array}{c}
s \\
\tan \alpha
\end{array}\right)
$$

and the resulting tyre force vector is

$$
\left(\begin{array}{c}
F_{x} \\
F_{y}
\end{array}\right)=P(|\boldsymbol{k}| ; B, C, D, E) \frac{F_{p}}{|\boldsymbol{k}|}\left(\begin{array}{l}
k_{x} \\
k_{y}
\end{array}\right)
$$

A typical set of resulting forces is shown in Fig. 14. 


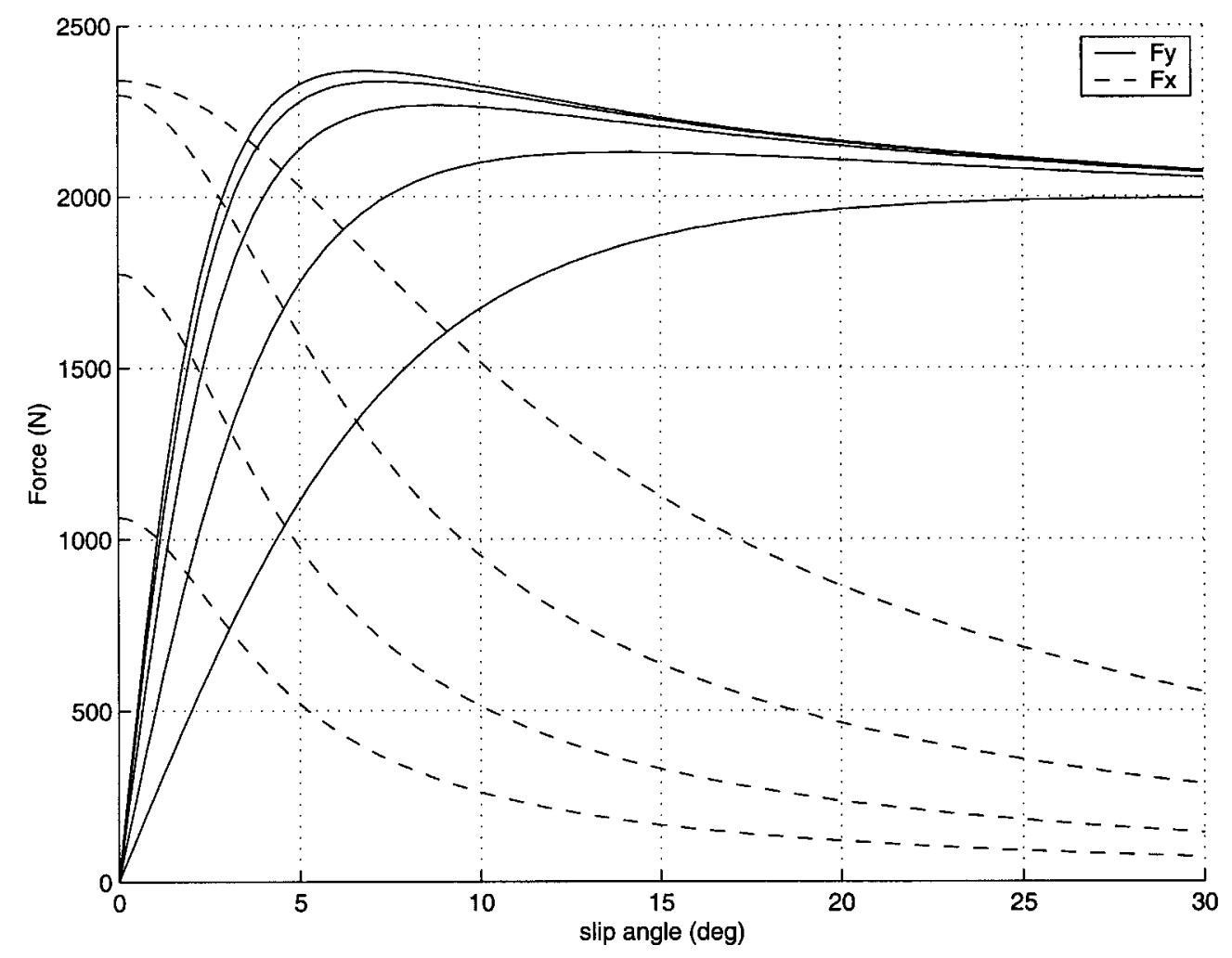

Fig. 14 Tyre forces at $2.5 \mathrm{kN}$ vertical load with variable longitudinal slip $(s=0.00,0.02,0.04,0.08,0.16)$

The final aspect of the control and simulation model is the transformation of drive/brake demand into longitudinal tyre slip. Rear wheel drive is assumed, with equal torque split between the wheels, while braking torque is split in the ratio 60:40 between the front and rear axles. The vehicle acceleration demand $u_{1}(t)$ is split according to these features, then multiplied by the vehicle mass $M$ to provide an individual wheel force demand $F_{x}^{\text {com }}$. In reality, this then enters into a complex low-level dynamic interaction, involving wheel rotation, driveline flexibility, a variety of rotary inertias, combustion lags, brake system mechanical properties, etc., as well as the transient build-up of forces at the road/tyre contact path. Time constants vary with gear or clutch state, and a large number of physical parameters influence the detailed response. In line with the approach adopted here, the simplest meaningful dynamic representation is adopted in the form of a first-order system:

$$
\dot{s}=\tau_{4}^{-1} C_{\alpha}^{-1}\left(F_{x}^{\mathrm{com}}-F_{x}\right)\left(1-\sigma|s| s_{\max }^{-1}\right)
$$

where $\sigma$ is short for the switching term [cf. equation (57)]

$$
\sigma=\sigma\left[s\left(F_{x}^{\mathrm{com}}-F_{x}\right)\right]
$$

The above equations allow the tyre force to track the commanded input and also incorporate a peak longitudinal slip (here $s_{\max }=0.5$ ), hence providing a simple form of antilock braking and traction control. Finally, in addition to the force limits inherent in the tyre model, an engine power limit is also imposed; for each of the rear wheels,

$$
F_{x}^{\text {com }}=\frac{W^{\max }}{2 U} \quad \text { when } u_{1} U>W^{\max }
$$

\section{SIMULATED SYSTEM PERFORMANCE}

A relatively brief set of simulations will now be presented, with the aim of testing the feasibility of the vector field approach and assessing how close the more realistic vehicle can come to emulating the behaviour of the idealized 'particle' response of Section 4. This is crucial, since if such behaviour is far from being comparable, it might be concluded that the earlier work is not very informative and that the vector field properties are of limited significance. If, on the other hand, some comparable behaviour is obtained, measures such as the maximum flow curvature and convergence eigenvalue are established as key features of the vehicle control problem. Implicit in this is the assumption that the vehicle controller for steering, brakes and throttle is operating effectively.

Even for a simple model, vehicle friction limits are determined by a variety of factors. Rather than consider these in detail, a rough estimate can be obtained by initially setting random throttle, brake and steering inputs that are of sufficient amplitude to exercise the vehicle at its handling limits. The inputs were chosen as 
'white noise' signals, band limited via a zero-order hold of $0.5 \mathrm{~s}$. Simulation of $20 \mathrm{~s}$ from an initial vehicle speed of $20 \mathrm{~m} / \mathrm{s}$ gave a peak vehicle acceleration magnitude of $8.5 \mathrm{~m} / \mathrm{s}^{2}$, which, although slightly in excess of the earlier assumed limit, gives a desirable small positive margin of error. For reference, the pseudo-random inputs were generated via the Simulink [11] block 'band limited white noise', using two-channel output and with noise power set at 1 and 0.1 for longitudinal and lateral controls respectively; the default seed was chosen for the pseudo-random number generator.

Using the same track section and reference vector field as in Fig. 5 and starting at the track centre with an initial velocity of $20 \mathrm{~m} / \mathrm{s}$ parallel to the centre-line, the vehicle path accurately tracks the vector field, giving a negligible deviation from the convergent path; therefore in this initial test the overall vehicle controller is certainly effective. Figure 15 shows the associated vehicle accelerations, resolved parallel and perpendicular to the mass centre velocity. As would be expected, nearly all of the acceleration is in the lateral direction, the small longitudinal acceleration being associated with a fluctuation in the vehicle speed, which occurs due to transient in the vehicle slip angle as the track curvature changes direction. From Fig. 16 it can be seen that the vehicle speed remains within $\pm 0.2 \mathrm{~m} / \mathrm{s}$ of the target, even though the vehicle slip angle varies across a range of around $\pm 4^{\circ}$. The control inputs to the vehicle are also shown in Fig. 16. The relatively small adjustments to longitudinal demand are somewhat exaggerated in the plot, and over- all there is a well-controlled steering action, albeit including a low-amplitude component at around $6 \mathrm{~Hz}$ which appears to be due to a dynamic interaction between the controller and the vehicle. From this, it can be concluded that the control is operating effectively, even though it may be capable of further refinement.

The control action can be more directly related back to the ideal control analysis of Section 3. Although the precise strategy defined in equation (23) has not been implemented, it would be hoped that the present control achieves the same general stability property of maintaining the scalar product $\boldsymbol{e} \cdot \dot{\boldsymbol{e}}=e \dot{e}$ of equation (24) to be as negative as possible. Figure 17 plots this 'stability product' throughout the simulation, and it is seen that apart from two short excursions around $t=4 \mathrm{~s}$ and $8 \mathrm{~s}$, the control works well to maintain the desired negativity.

Another area of comparison can be seen in the set of vehicle trajectories resulting from entry positions ranging across the track (Fig. 18). This is precisely analogous to Fig. 7 and shows similarly excellent path tracking for all left and central entry points. For right-hand bias the lateral control initially appears superior to that of Fig. 7, but this is counteracted by the greater deviations experienced on the second curve. Speed control is similar in both examples, with some slowing down experienced from the $20 \mathrm{~m} / \mathrm{s}$ target when entering from right of centre; in both cases the worst speed reduction occurs with far right entry, minimum values being 15.2 and $14.4 \mathrm{~m} / \mathrm{s}$ for Figs 7 and 18 respectively.

A number of other simulations have been conducted,

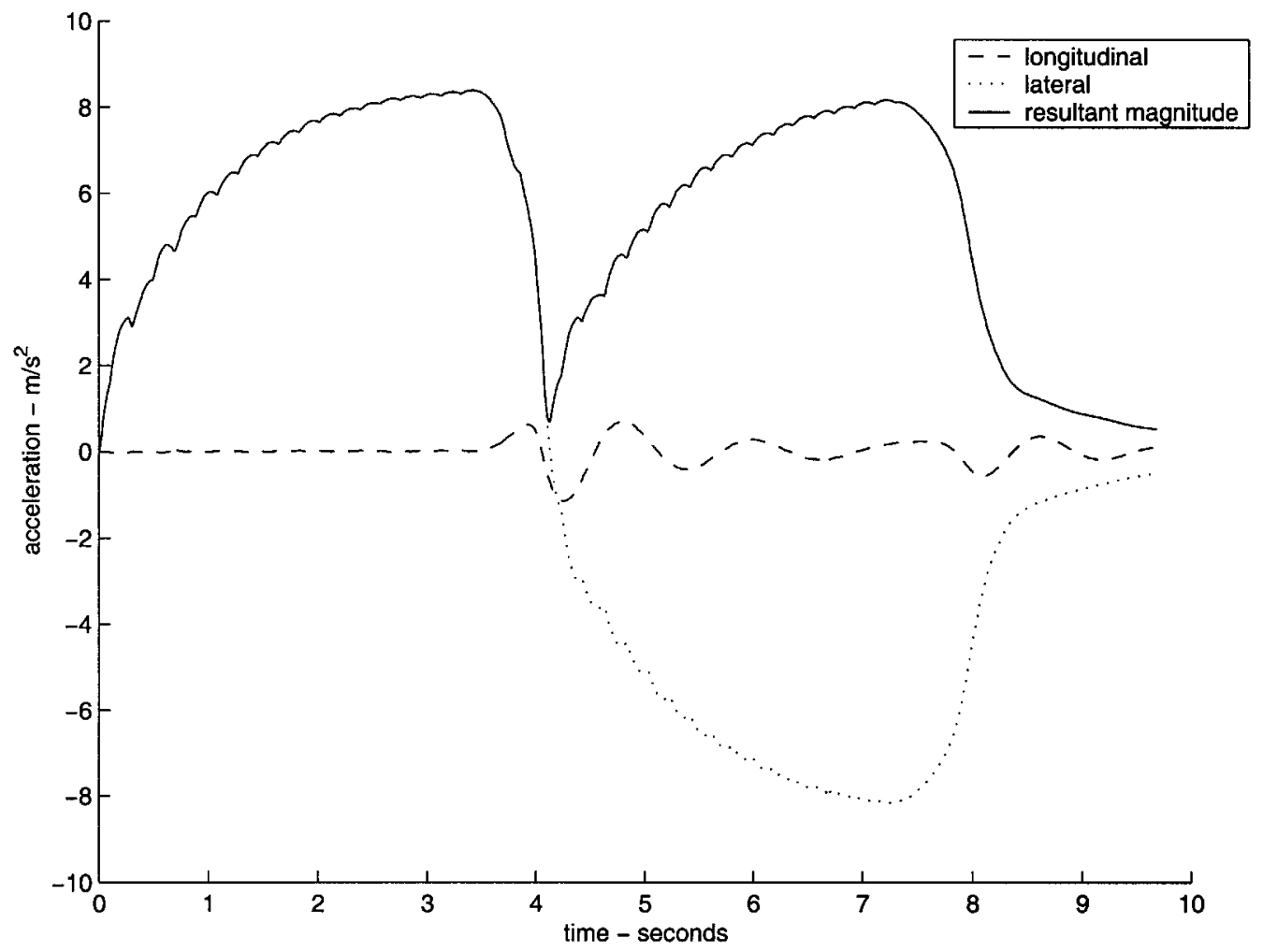

Fig. 15 Vehicle accelerations 

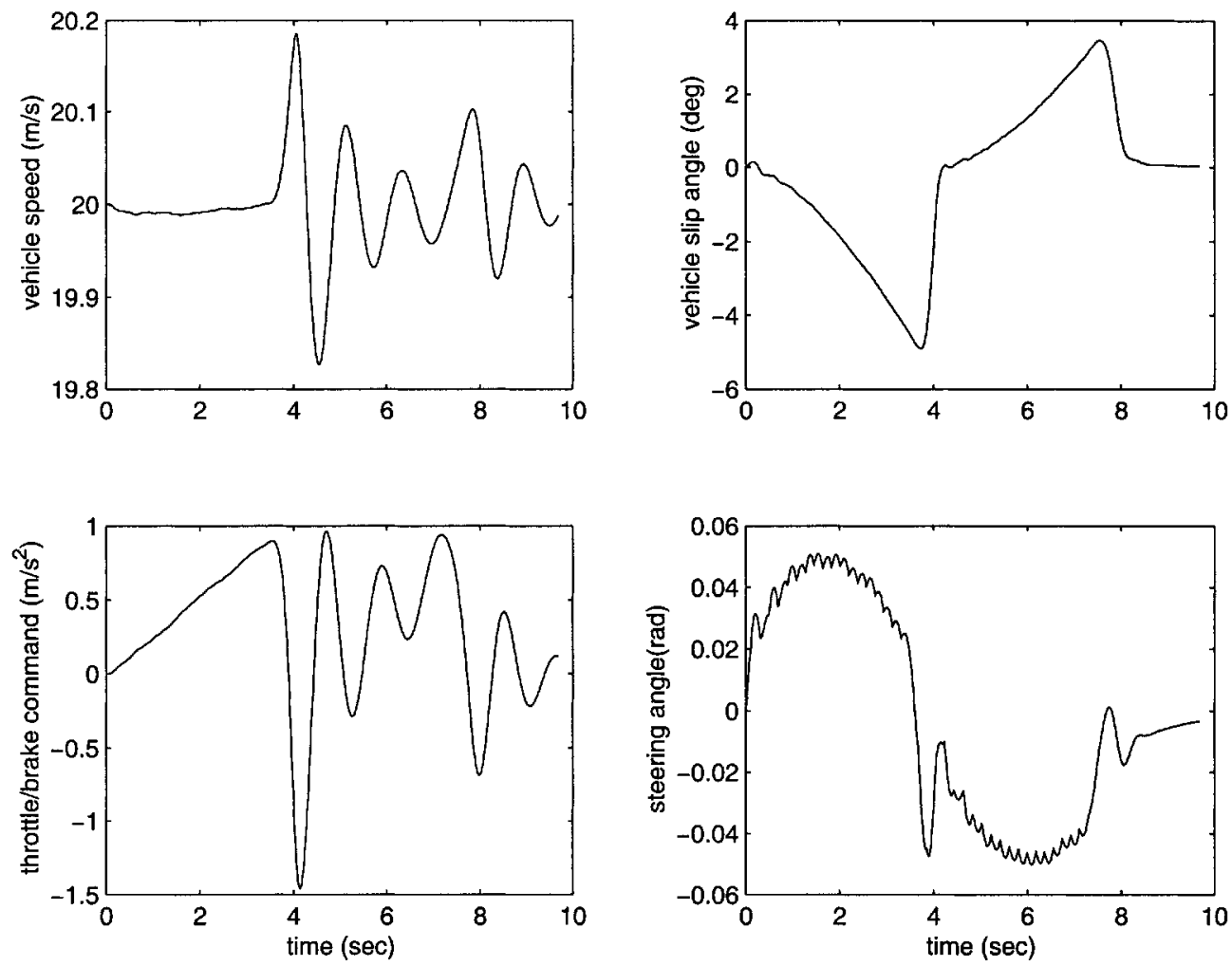

Fig. 16 Vehicle speed, slip angle and controller outputs

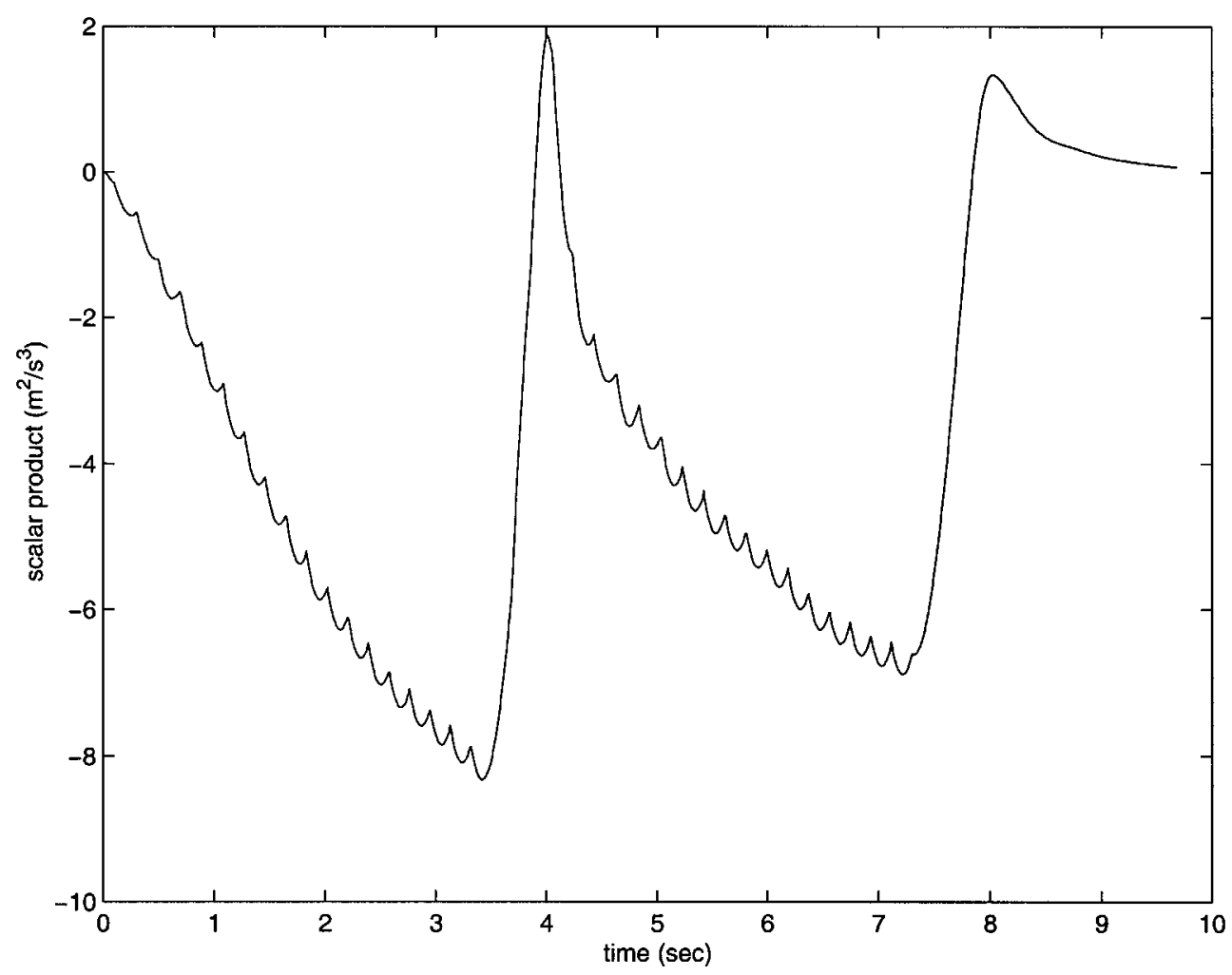

Fig. 17 Stability product in the vehicle control simulation 


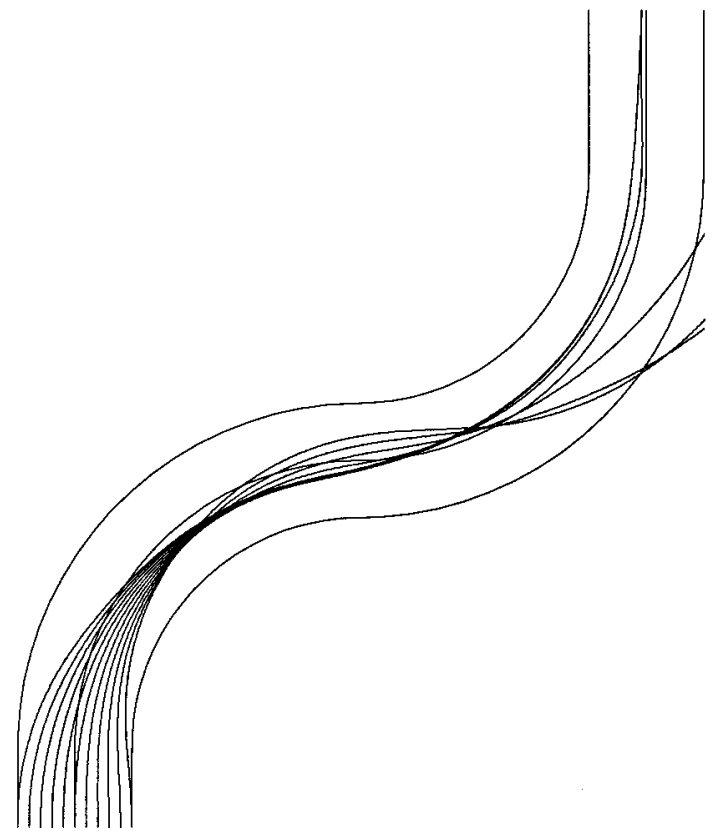

Fig. 18 Vehicle paths in the control simulation

and generally the vehicle responds in a predictable and controlled manner, though as in real life, limitations of the vehicle set-up occasionally show. For example, in one test the vehicle was given an initial velocity of $20 \mathrm{~m} / \mathrm{s}$ along the $y$ axis, while the reference field was a uniform $20 \mathrm{~m} / \mathrm{s}$ parallel to the $x$ axis. Vehicle speed was maintained within $\pm 0.5 \mathrm{~m} / \mathrm{s}$, while full steering lock was quickly reached, giving rise to significant understeer, the lateral acceleration only achieving $5 \mathrm{~m} / \mathrm{s}^{2}$. In other cases, the vehicle is seen to generate oversteering behaviour, but the yaw response is well damped by the automated driver system. Clearly, there are many further studies that could be conducted from this point onwards, including making changes to the vehicle set-up and extending the number of degrees of freedom in the vehicle model. However, it is felt that the main point has already been demonstrated, namely that the overall vehicle control can successfully approximate that of the ideal frictionlimited vehicle, and hence there is significant relevance in the vector field results obtained earlier.

\section{CONCLUSIONS}

A reference vector field has been used to develop a new conceptual framework for autonomous vehicle control. For any specific implementation, the automated driver clearly only requires local information, in the form of $w$ and its derivatives $\nabla \boldsymbol{w}$, so the method of using the vector field as a complete ('distributed parameter') entity in the control task could be seen as being overstated in the foregoing. A corresponding narrow view of what has been achieved would then be as follows. Firstly, a simple particle-based model of the vehicle dynamics has been used to derive sufficiency criteria for convergence under friction-limited vehicle control. In this, the flow acceleration and flow convergence of the reference field have been shown to be of equal significance, and this is probably sufficient to justify the use of vector field language in the development of the automated driver. Secondly, a number of field construction methods have been investigated and assessed in terms of these criteria, although the investigation has been largely illustrative and far from exhaustive. Thirdly, the framework of control based on the reference field has been extended to the control of a more realistic vehicle, albeit using relatively simple controllers and a somewhat idealized vehicle representation. The automated driver has the ability to work successfully at the handling limits of the vehicle, applying sometimes complex steering, braking and throttle inputs to the vehicle, to achieve responses that are quite similar to the ideal 'particle' responses.

The underlying methodology presented in the paper is inherently based on non-linear dynamics and large disturbances. Such scope appears essential for integrating discrete actions (e.g. collision avoidance) into the framework of autonomous vehicle control. In this scenario, once a potential collision has been detected, a higher level decision could be made to switch the reference field away from the obstacle, so that simultaneous steering and braking are employed in a structured and predictable manner. From the current perspective, it seems that the relevant concept is once again the reference vector field as a whole, rather than simply the instantaneous values being passed to the controller.

More generally, the main potential strengths of the vector field approach are that it provides a global framework suitable for analysing general properties of stability and robustness, and offers a natural modular structure leading to improved insight and scope for flexible and adaptive implementation. This is not to imply that the resulting specific control system performance is inherently better than can be achieved by more traditional formulations; indeed, any approach, such as Model Predictive Control [7], that invokes direct numerical optimization should in a narrow sense outperform any other controller, including that presented here.

It has been brought to the authors' attention that a control technique based on velocity field concepts has been independently proposed by $\mathrm{Li}$ and Horowitz $[12,13]$ for the control of robotic manipulators. There is a strong similarity in that they propose a velocity field to be designed a priori to 'encode' for a target contour, with the field convergent to the desired path. The usefulness of a field approach is once again that global convergence and robustness criteria can be deduced, and there is no restriction to considering linear models and 'small deviations'. In the general area of robotic manipulators, the configuration space takes the form of a differentiable manifold of potentially high dimension, and much of $\mathrm{Li}$ and Horowitz's work deals with the mathematical analy- 
sis of the differential geometry involved. The velocity field plays an essential role in their analysis, but is not related directly to control system performance, e.g. in terms of the convergence properties of the defined field. In place of the limited accelerations considered here, the robotics problem assumes limited energy resources for the manipulator. Furthermore, Li and Horowitz's velocity field is defined in the full dimension of the configuration space, as opposed to the reduced dimensionality considered here for the vehicle motion in the plane.

Returning to the simulated performance of the fieldbased automated driver for vehicle dynamics, a general indication of the capability of the controller comes from an unconventional source, namely its use in a computer game [1]. Here multiple vehicles-'competitors' and general 'traffic'-operate simultaneously under the control of independent automated drivers of the type described, and can be seen operating in a virtual environment involving complex large-angle vehicle dynamics and including three-dimensional geometry of roads and scenery, as well as collision dynamics with other vehicles. Each vehicle simulation model employs Newton-Euler equations for the six-degree-of-freedom rigid vehicle body motions, linearized suspension geometry (thus including effects of suspension roll moments and jacking under cornering, anti-pitch behaviour under braking, etc.), powertrain and braking systems dynamics and a combined-slip tyre model with load-dependent friction limits. The model was designed for efficient real-time simulation and is described in detail in reference [14]. From viewing the game simulation, it appears that a controlled recovery is normally achieved after large disturbances such as a collision between two vehicles. Clearly no formal conclusions can be drawn from this 'experiment', but it further suggests that an automated driver based on the approach presented here might provide a robust and flexible approach to the automation of a complex and realistic driving task.

Finally, the work presented above offers significant scope for further development:

1. Reference field adaptation. There had been an implicit assumption that directional information is completely independent of vehicle states; the overall goal is essentially the same, whether the vehicle is at rest on the wrong side of the track or driving at maximum speed on the racing line. Up to a point this appears reasonable; e.g. the reference field would not be expected to alter because of, say, a transient excursion in the yaw velocity. However, taken too literally, the goals would remain the same even if the brakes fail or a tyre deflates, but clearly in these cases the need for adaptation of the reference field becomes necessary. Similarly, reference field adaptation is needed to cope with multi-vehicle interactions, collision avoidance, etc.

2. Variable friction circle. A constant friction circle has been assumed, whereas in reality this varies with aerodynamic downforce and local variations in surface friction and vertical track geometry; such factors can be incorporated quite naturally into the vector field description.

3. Reference field optimization. There is considerable scope for optimization of the reference field, taking into account the relative stability of the convergent path, but without involving the complexity of detailed vehicle dynamics. It is also plausible to extend the approach to the optimization of lap times in a way that incorporates the essential detailed dynamics of the vehicle.

4. Assessment of transient vehicle dynamics. Based on the theoretical aspect of the formal convergence criteria, it appears natural to assess transient vehicle dynamic performance in terms of how well a real vehicle can satisfactorily approximate the ideal friction-limited 'particle' (see Section 6). This may, for example, form a basis for future systematic performance analysis of active chassis control systems.

\section{REFERENCES}

1 Clockwork Games Limited, Vanishing Point (interactive video game for Sony Playstation), 2001 (Acclaim, New York).

2 Weir, D. H. and McRuer, D. T. Dynamics of driver vehicle steering control. Automatica, 1970, 6, 87-98.

3 Tomizuka, M. and Whitney, D. E. The human operator in manual preview tracking (an experiment and its modeling via optimal control). Trans. ASME, J. Dynamic Systems, Measmt and Control, 1976, 98, 407-413.

4 Modjtahedzadeh, A. and Hess, R. A. A model of driver steering control behavior for use in assessing vehicle handling qualities. Trans. ASME, J. Dynamic Systems, Measmt and Control, 1993, 115, 456-464.

5 Horiuchi, S. and Yuhara, N. An analytical approach to the prediction of handling qualities of vehicles with advanced steering control system using multi-input driver model. Trans. ASME, J. Dynamic Systems, Measmt and Control, 2000, 122, 490-497.

6 Sharp, R. S., Casanova, D. and Symonds, P. A mathematical model for driver steering control, with design, tuning and performance results. Veh. System Dynamics, 2000, 33, 289-326.

7 Prokop, G. Modeling human vehicle driving by model predictive online optimization. Veh. System Dynamics, 2001, 35, 19-53.

8 MacAdam, G. C. and Johnson, G. E. Application of elementary neural networks and preview sensors for representing driver steering control behaviour. Veh. System Dynamics, 1996, 25, 3-30.

9 Plöchl, M. and Lugner, P. A 3-level driver model and its application to driving simulations. Veh. System Dynamics, 1999, 33, 71-82.

10 Bakker, E., Nyborg, L. and Pacejka, H. B. A new tire model with an application in vehicle dynamics studies. SAE paper $890087,1989$. 
11 Matlab Version 5.3.1, Simulink Version 3.0, Sepember 1999 (The MathWorks Inc., Natick, Massachusetts).

12 Li, P. Y. and Horowitz, R. Passive velocity field control of mechanical manipulators IEEE Trans. Robotics and Automn, 1999, 15(4), 751-763.

13 Li, P. Y. and Horowitz, R. Passive velocity field control (PVFC): Parts I and II. IEEE Trans. Autom. Control, 2001, 46(9), 1346-1371.

14 Dixon, P. J. Sideslip tracking strategies for vehicle handling control. PhD thesis, Department of Aeronautical and Automotive Engineering, Loughborough University, 2002.

\section{APPENDIX}

\section{Fixed lookahead policy}

Figure 19 shows the construction of $\boldsymbol{w}(\boldsymbol{x})$ using a fixed lookahead policy, for a straight-line target path that coincides with the $x$ axis. From the figure,

$$
w(x, y)=\frac{U}{D}\left(\begin{array}{c}
L \\
-y
\end{array}\right)
$$

where a fixed speed $U$ is assumed and $D=\sqrt{y^{2}+L^{2}}$ is the distance from the vehicle mass centre to the target point on the track. The integral curves are hence defined by

$$
\frac{\mathrm{d} x}{\mathrm{~d} t}=\frac{U L}{D}, \quad \frac{\mathrm{d} y}{\mathrm{~d} t}=\frac{-U y}{D}
$$

or in non-parametric form,

$$
\frac{\mathrm{d} y}{\mathrm{~d} x}=\frac{-y}{L}
$$

Integration of this differential equation is elementary, yielding

$$
y=y_{0} \exp \left[\frac{-\left(x-x_{0}\right)}{L}\right]
$$

The path acceleration $\kappa$ is easily found by differentiation of equation (69) to give

$$
\kappa=|(\boldsymbol{w} \cdot \nabla) \boldsymbol{w}|=\frac{U^{2} L y}{D^{3}}
$$

which clearly tends to zero as $y \rightarrow 0$ and also as $y \rightarrow \infty$ (in the latter case $D \rightarrow \infty$ also). It is then interesting though elementary to show that $\kappa$ achieves a unique

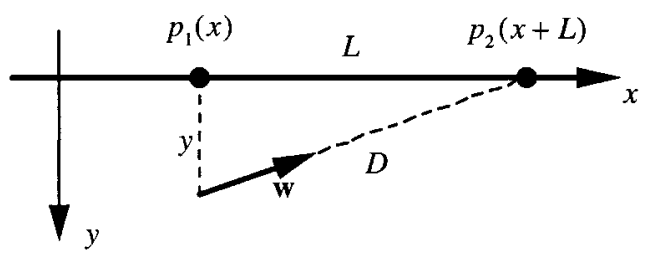

Fig. 19 Fixed lookahead to a straight line reference path maximum when

$$
y=\frac{L}{\sqrt{2}}
$$

with peak acceleration

$$
\kappa^{\max }=\frac{2 U^{2}}{3 \sqrt{3} L}
$$

Also, from equation (69) the divergence matrix (9) evaluates to

$$
\mathbf{H}=-\frac{U L}{2 D^{3}}\left(\begin{array}{cc}
0 & y \\
y & 2 L
\end{array}\right)
$$

which has eigenvalues

$$
\mu=-\frac{U L(L \pm D)}{2 D^{3}}
$$

Hence the least eigenvalue is

$$
\mu_{1}=-\frac{U L(L+D)}{2 D^{3}}=-\frac{U}{2 L}\left(\xi^{3}+\xi^{2}\right)
$$

where $\xi=L / D$ uniquely defines the dependence on $y$. Since $0<\xi \leqslant 1$, it is clear that $\mu_{1}$ attains its greatest magnitude at $\xi=1$, which occurs on the track itself $(y=0)$, and with value

$$
\mu_{1}^{\min }=-\frac{U}{L}
$$

\section{Geometrical interpretation of the divergence matrix}

Figure 20 depicts the local geometry as two nearby points $\boldsymbol{x}_{1}(t)$ and $\boldsymbol{x}_{2}(t)$ are transported along the $\boldsymbol{w}$ streamlines to $\boldsymbol{x}_{1}(t+\Delta t)$ and $\boldsymbol{x}_{2}(t+\Delta t)$ respectively. The initial separation distance $d(t)=\left|\boldsymbol{x}_{2}(t)-\boldsymbol{x}_{1}(t)\right|$ is modified by the divergence of the flow lines; differentiating the square of the separation distance gives

$$
\begin{aligned}
2 d \dot{d} & =2\left(x_{2}-x_{1}\right) \cdot\left(\dot{x}_{2}-\dot{x}_{1}\right) \\
& =2 d \boldsymbol{n} \cdot\left(w\left(x_{2}\right)-w\left(x_{1}\right)\right)
\end{aligned}
$$

where $\boldsymbol{n}$ is the unit vector pointing from $\boldsymbol{x}_{1}(t)$ to $\boldsymbol{x}_{2}(t)$. Hence

$$
\dot{d}=n \cdot\left(w\left(x_{2}\right)-w\left(x_{1}\right)\right)
$$

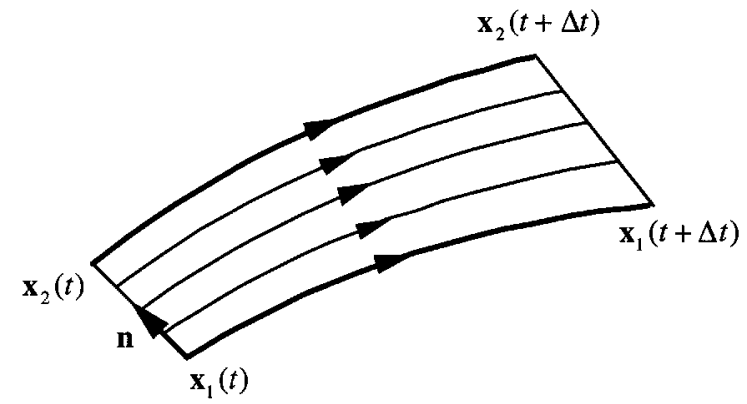

Fig. 20 Local effect of flow divergence 


$$
=\boldsymbol{n} \cdot\left(d(\boldsymbol{n} \cdot \nabla) \boldsymbol{w}\left(\boldsymbol{x}_{1}\right)+O\left(d^{2}\right)\right)
$$

where a Taylor series has been used to approximate the finite difference. Hence, implicitly at point $\boldsymbol{x}_{1}$,

$$
\begin{aligned}
\lim _{d \rightarrow 0} \frac{\dot{d}}{d} & =\boldsymbol{n} \cdot((\boldsymbol{n} \cdot \nabla) \boldsymbol{w}) \\
& =\sum_{i} \sum_{j} n_{i} n_{j} \frac{\partial w_{i}}{\partial x_{j}} \\
& =\frac{1}{2} \sum_{i} \sum_{j} n_{i} n_{j}\left(\frac{\partial w_{i}}{\partial x_{j}}+\frac{\partial w_{j}}{\partial x_{i}}\right)
\end{aligned}
$$

where summation has allowed the expression in parentheses to be made symmetric. Hence, from equation (9),

$$
\lim _{d \rightarrow 0} \frac{\dot{d}}{d}=\boldsymbol{n}^{\mathrm{T}} \mathbf{H} \boldsymbol{n}
$$

Now let $v_{1}$ and $v_{2}$ be the (orthonormal) eigenvectors of $\mathbf{H}$ corresponding to $\mu_{1}$ and $\mu_{2}$, with $\mu_{1} \leqslant \mu_{2}$. If

$$
\boldsymbol{n}=n_{1} \boldsymbol{v}_{1}+n_{2} \boldsymbol{v}_{2}, \quad n_{1}^{2}+n_{2}^{2}=1
$$

then

$$
\begin{aligned}
\lim _{d \rightarrow 0} \frac{\dot{d}}{d} & =\mu_{1} n_{1}^{2}+\mu_{2} n_{2}^{2} \\
& \geqslant \mu_{1} n_{1}^{2}+\mu_{1} n_{2}^{2} \\
& =\mu_{1}
\end{aligned}
$$

which proves equation (11) as required. Note that if $\boldsymbol{n}_{1}$ and $\boldsymbol{n}_{2}$ define the sides of a small rectangular area, with sides of length $d_{1}$ and $d_{2}$, the above easily leads to the result

$$
\frac{\dot{A}}{A} \equiv \frac{(\mathrm{d} / \mathrm{d} t)\left(d_{1} d_{2}\right)}{d_{1} d_{2}}=\frac{\dot{d}_{1}}{d_{1}}+\frac{\dot{d}_{2}}{d_{2}}=\mu_{1}+\mu_{2}
$$

i.e. the sum of the eigenvalues determines the area divergence (which becomes volume divergence in three dimensions).

\section{Differential inequality for path convergence}

Referring to equation (42),

$$
\dot{D}(t) \leqslant-\phi(D)+\psi(t)
$$

where $D \geqslant 0$ and $\psi(t)$ and $\phi(D)$ are non-negative functions with properties given in equations (36) and (40) respectively. $\phi(D)$ is monotonically non-decreasing, so that

$$
D_{1}<D_{2} \Rightarrow \phi\left(D_{1}\right) \leqslant \phi\left(D_{2}\right)
$$

Also, from the properties of $\psi(t)$, given any $D_{0}>0$, there exists $t_{0}>0$ such that

$$
t \geqslant t_{0} \Rightarrow \psi(t) \leqslant \frac{1}{2} \phi\left(D_{0}\right)
$$

Then if $t>t_{0}$ and $D \geqslant D_{0}$, from equation (84)

$$
\begin{aligned}
\dot{D}(t) & \leqslant-\phi(D)+\frac{1}{2} \phi\left(D_{0}\right) \\
& \leqslant-\phi\left(D_{0}\right)+\frac{1}{2} \phi\left(D_{0}\right)
\end{aligned}
$$

i.e.

$$
t \geqslant t_{0}, \quad D \geqslant D_{0} \Rightarrow \dot{D} \leqslant-\frac{1}{2} \phi\left(D_{0}\right)
$$

From this it can be shown that there always exists a time $t_{1} \geqslant t_{0}$ for which $D\left(t_{1}\right)<D_{0}$. There are three cases:

1. If $D\left(t_{0}\right)>D_{0}$, then from the minimum gradient in (87) there is a finite time $t$ at which $D(t)=D_{0}$, and again the gradient condition ensures $D\left(t_{1}\right)<D_{0}$, for $t_{1}=t+\delta t$, with some small time increment $\delta t$.

2. If $D\left(t_{0}\right)=D_{0}$, then, as above, choose $t_{1}=t_{0}+\delta t$.

3. If $D\left(t_{0}\right)<D_{0}$, then choose $t_{1}=t_{0}$.

It is also impossible for $D(t)$ to subsequently increase sufficiently to exceed the specified value $D_{0}$. Such an increase would imply $D\left(t_{2}\right)=D_{0}$ for some $t_{2} \geqslant t_{0}$, with $\dot{D}\left(t_{2}\right)>0$, and this clearly contradicts the negative derivative condition in (87). Hence there is always some time $t_{1} \geqslant 0$ after which $D(t)$ remains bounded by $D_{0}$. Since $D_{0}$ may be chosen arbitrarily small, this proves the assertion that $D(t) \rightarrow 0$ as $t \rightarrow \infty$. 